\title{
Neurophénoménologie du signe linguistique : Apport du modèle Phénoménologique Morphodynamique et Structuraliste (PMS) à la compréhension des mécanismes neuraux sous-tendant la donation de sens
}

\author{
Jean VION-DURY*, Mireille BESSON*, Michel CERMOLACCE*, \\ Daniele SCHÖN ${ }^{\diamond} \&$ David PIOTROWSKI*
}

\begin{abstract}
RÉSUMÉ. Cet article décrit un programme de recherche en neurosémiotique. L'idée principale consiste à proposer la composante N400 du potentiel évoqué comme «arbitre empirique » entre deux paradigmes de donation de sens: le paradigme fonctionnaliste des sciences cognitives, décrivant la séquence d'accès au lexique, et un paradigme réunissant le structuralisme saussurien, la phénoménologie husserlienne et la morphodynamique de Thom et Petitot (paradigme morphodynamique et structuraliste, PMS). Le paradigme PMS assume la consubstantialité du signifiant et du signifié (Saussure), ainsi que les diverses modalisations de la conscience verbale, dans un modèle dynamique, sans séquence d'accès lexical, mais en faisant intervenir des attracteurs relatifs au sens. La composante N400 est alors proposée pour mettre à l'épreuve de manière empirique (neuroscientifique) le paradigme PMS, celui-ci étant de nature à résoudre certaines anomalies empiriques que ne peut expliquer le paradigme cognitif d'accès au lexique.
\end{abstract}

Mots-clés : Potentiels évoqués, EEG, N400, phénoménologie, Husserl, structuralisme, Saussure, accès lexical, sciences cognitives, morphodynamique.

Abstract. Neurophenomenology of the Linguistic Sign: Contribution of the Phenomenological Morphodynamics and Structuralist (PMS) Model to Understanding the Neural Mechanisms Underlying the Constitution of Meaning. This paper describes a research program in neurosemiotics. The main idea is to propose the N400 component of the Event-Related Potential (ERP) as an «empirical referee » between two paradigms aiming at understanding meaning constitution. The first one is the classic functionnalist paradigm of cognitive sciences that describes computations for lexical access. The second one gathers Saussure structuralism, Husserlian phenomenology and Thom and Petitot morphodynamics (phenomenology,

* Unité de Neurophysiologie, Psychophysiologie et Neurophénoménologie, Pôle Universitaire de Psychiatrie - Laboratoire de Neurosciences Cognitives (LNC), UMR CNRS 7291, Aix-Marseille Universit. Jean.VION-DURY<at>ap-hm.fr.

" Laboratoire de Neurosciences Cognitives (LNC), UMR CNRS 7291, Aix-Marseille Université. mireille.besson<at>amu-univ.fr.

* Unité de Neurophysiologie, Psychophysiologie et Neurophénoménologie, Pôle Universitaire de Psychiatrie - Laboratoire de Neurosciences Cognitives (LNC), UMR CNRS 7291, Aix-Marseille Université. Michel.CERMOLACCE<at>ap-hm.fr.

${ }^{\diamond}$ Institut des Neurosciences des Systèmes, (ISC) UMR INSERM 1106, Aix-Marseille Université Laboratoire de Neurosciences Cognitives (LNC), UMR CNRS 7291, Aix-Marseille Université. daniele.schon<at>univ-amu.fr.

- Linguistique, Anthropologie et sociolinguistique (LIAS), IMM CNRS/EHESS 8178, Paris.

david.piotrowski<at>ehess.fr. 
morphodynamics and structuralism, PMS paradigm). PMS assumes the co-presence of signified and signifier (Saussure), and the various modalisations of verbal consciousness (Husserl) together within a dynamics model involving meaning attractors without lexical access. The N400 component is proposed to empirically challenge the PMS paradigm that can help solve some empirical abnormalities that cannot be explained by the cognitive paradigm of lexical access.

Keywords: Event-related potentials, EEG, N400, phenomenology, Husserl, structuralism, Saussure, lexical access, cognitive sciences, morphodynamics.

\section{I - INTRODUCTION}

Cet article se veut programmatique d'une proposition de recherche pluridisciplinaire visant à réaliser une mise à l'épreuve par la neurophysiologie d'un modèle linguistique phénoménologique, morphologique et structuraliste qui concerne les processus de donations de sens des objets de nature sémiotique ${ }^{1}$. Le potentiel évoqué N400 sera alors proposé pour tenter de saisir les mécanismes neuraux sous-tendant la donation de sens.

Dans cet article nous serons amenés à prendre en compte les conséquences épistémiques de la «consubstantialité » du signifiant et du signifié, c'est-à-dire de la nature indivise du signe ${ }^{2}$, qui constitue un point d'appui partagé par les problématiques husserlienne et saussurienne. Pour ce faire, nous avons choisi une voie étroite qui passe d'une part par la conception Husserlienne du signe et, d'autre part, par la morphodynamique structurale du signe saussurien.

Le modèle proposé et que nous qualifierons de phénoménologique, morphodynamique et structuraliste (PMS) se fonde sur trois socles : 1) l'unité constitutive a priori de la forme et du sens (versus une approche intégrative $a$ posteriori de la forme et du sens); 2) la différentialité du sens ${ }^{3}$ reprise de Saussure, modélisée par l'approche qualitative des systèmes dynamiques, et 3 ) une approche phénoménologique qui reconnait des phases de conscience verbale intermédiaires entre le son et le sens et qui soutient une approche conscientielle de la linguistique.

Nous examinerons donc d'abord en détail le contenu de ces trois socles qui soutiennent une perspective relativement inhabituelle qui contraste avec les

\footnotetext{
${ }^{1}$ Le sémantique concerne le sens dans ses régimes de formations et d'individuation, et le sémiotique concerne le signe dans son fonctionnement dans un système. On retrouve ici l'opposition sémiotique/ sémantique de Benveniste, où le sémiotique renvoie au signe comme partie du système, et le sémantique au sens qu'actualise le signe en discours (Benveniste, 1976).

${ }^{2}$ Soit l'on envisage l'unité indivise du signe comme un phénomène parmi d'autres et passible d'un même traitement explicatif ou comme principe explicatif d'analyses empiriques, soit on l'envisage comme donnée originaire et matière d'un questionnement premier. C'est ce caractère central de cette «mystérieuse » interpénétration du son et du sens dont nous cherchons à approcher le principe et les formes internes. Car « [La] notion de renvoi, [présente] dans le couple saussurien signifiant-signifié ou dans le couple glossématique expression-contenu, [est] un indéfinissable [...] qui constitue, croyons nous, l'un des points frontières entre linguistique et philosophie du symbolisme. Accepter cette notion comme primitive, c'est se placer sur le versant où opère le linguiste ; tenter d'approfondir l'idée de renvoi, c'est passer délibérément du côté du philosophe, et renoncer à construire des modèles de ce qui est. Le linguiste partira nécessairement du constat de l'indissoluble liaison du signifiant et du signifié comme définition même du signe » (Granger, 1979, p. 205).

${ }^{3}$ Le sens de A est tout ce que B n'est pas.
} 
théories fonctionnalistes cognitivistes classiques et plus particulièrement avec les approches modulariste ou computationnelle.

Dans un second temps notre intention est d'interpréter à partir de cette approche phénoménologique et structuraliste du langage quelques résultats expérimentaux qui, à la lumière conceptuelle du paradigme (Kuhn, 2008) « classique» des sciences cognitives et de la neurolinguistique, font figure d'anomalies. Ces résultats expérimentaux concernent la composante N400 du potentiel évoqué qui compte parmi les effets électro-encéphalographiques (EEG) les plus robustes et surtout les plus étudiés en neurolinguistique.

Puis nous montrerons comment le modèle PMS serait à même de mieux rendre compte de ces anomalies, en envisageant un protocole neurolinguistique qui reste à conduire dans la suite de ce projet.

\section{II - SAUSSURE ET HUSSERL : UNE COMMUNAUTÉ DE VUES}

Il est intéressant de remarquer qu'entre 1907 et 1911 à la fois Saussure et Husserl ont élaboré une théorie du signe qui acte de la coappartenance du signifiant et du signifié, l'un ne pouvant être détaché de l'autre. Autrement dit, pour Saussure comme pour Husserl le signe a une nature indivise, et, reprenant ici les termes de Benveniste (1976), ses deux faces sont consubstantielles.

\section{II.1 - La substance commune du signifiant et du signifié}

Comme l'indique Saussure dans son cours de linguistique générale, «le signe linguistique unit non une chose et un nom, mais un concept et une image acoustique. Cette dernière n'est pas le son matériel, chose purement physique, mais l'empreinte psychique de ce son, la représentation que nous en donne le témoignage de nos sens [...]. Le signe linguistique est donc une entité psychique à deux faces [...]. Ces deux éléments sont intimement unis et s'appellent l'un l'autre. Nous appelons signe la combinaison du concept et de l'image acoustique. Nous proposons de conserver signe pour désigner le total et de remplacer concept et image acoustique respectivement par signifié et signifiant » (Saussure, 1995, p. 98-99).

Cette définition du signe a fait et fait encore l'objet de débats dans la communauté linguistique (Klinkenberg, 2000, p. 92-112). L'objet de la discorde relève de cette double face du signe saussurien : à la fois signifiant et signifié, image acoustique et concept. Le problème est le «à la fois ». Plutôt qu'ambiguïté, nous verrons que le problème fondamental réside dans la « consubstantialité » du signifiant et du signifié : leur incorporation réciproque.

\section{II.2 - Le signe «assemblage » ou signe indicatif}

Husserl comme Saussure distingue le « vrai » signe, linguistique, de teneur indivise, de celui résultant d'un simple «assemblage » d'un symbole et d'une idée comme il en est du signe « conventionnel ». Ce dernier signe, Husserl le dénomme «indicatif » et le décrit comme enchaînement de deux moments de conscience : il y a d'abord un certain vécu de conscience, qui est la perception de la marque symbolique, puis, par sa fonction constituante, le symbole 
réoriente la conscience vers un autre contenu qui est la chose, l'idée ou l'état de choses à communiquer dont on veut informer l'interlocuteur (Husserl, 2003, p. 29). C'est ce qui se passe pour une sonnerie de téléphone. Saussure définira le signe indicatif comme groupement son-idée.

\section{II.3 - Le «vrai » signe : le signe signitif}

Husserl et Saussure élaborent ainsi pareillement leur conception du « vrai » signe, le signe «signitif», «expression» pour l'un (Husserl), unité signifiant/signifié pour l'autre (Saussure). Tous deux considèrent que ce qui constitue l'essence du signe «authentique » est une sorte d'incorporation des faces du signe qui interdit d'aller vers l'un sans solliciter l'autre: «on ne saurait isoler ni le son de la pensée, ni la pensée du son; on n’y arriverait que par une abstraction dont le résultat serait de faire de la psychologie pure ou de la phonologie pure » (Saussure, 1917, p. 15). Pour Husserl, le signe signitif s'inscrit dans un seul et même moment de conscience : l'appréhension du divers sensible et son élaboration comme phénomène-signe s'opèrent dans un seul acte intentionnel qui est la visée d'un objet de « contenu ».

Alors que le symbole signifie en tant qu'il est « interprété » (Husserl, 2003, p. 36), l'expression signifie au «sens prégnant» du terme (Husserl, 2003, p. 30). En d'autres termes, le «vrai » signifiant, l' «expression», comporte dans son identité phénoménale même l'orientation de la conscience vers une signification. C'est «en vertu [des actes intentionnels que] l'expression est plus qu'un simple phénomène sonore [...] » (Husserl, 2003, p. 43).

En revanche, une architecture fonctionnaliste (cf. plus loin les approches fonctionnalistes et modularistes) organisée en niveaux de représentation indépendants et hiérarchisés conduit à interpréter le signe linguistique saussurien en signe « indicatif» ou « conventionnel ». La logique fonctionnaliste ne retient que la première partie de la définition du signe saussurien : les termes de la dualité indivise signifiant/signifié se trouvent rapportés respectivement aux formats autonomes de l'image acoustique et du concept. La «coappartenance » du signifiant et du signifié dans le signe est ainsi ignorée, mise de côté. La conception modulaire et hiérarchique permet ainsi de séparer le signifiant du signifié et de proposer plus aisément une construction séquentielle du signe.

\section{II.4 - Difficulté à penser le signe linguistique}

Il y a quelque difficulté à penser cette essence indivise. D'où de nombreux recours aux métaphores pour en approcher la teneur ou en exprimer le principe. Ainsi, on va parler de la «consubstantialité » des faces du signe, de leur «fusion», de leur «assimilation réciproque », de «symbiose (Benveniste, 1966, p. 51), de «dualisme indissoluble» du signe linguistique (Jakobson, 2003, p. 162), de «symphyse » (Guillaume, 1991, p. 247).

On pourrait arguer que la conception saussurienne du signe est une anomalie, une aberration conceptuelle, et, par-là, non opérante. Mais l'étonnante convergence des vues de Saussure et de Husserl quant au signe, l'un ayant en vue la langue et l'autre la conscience doit se contextualiser dans l'ambiance intellectuelle de l'époque, qui se posait la question de la nature 
ondulatoire (Maxwell, dans les années 1850-65) et/ou corpusculaire de la lumière (Plank, Einstein, Perrin, dans les années 1900-1905) sans encore les associer dans un même phénomène mais posant la question de la dualité ondecorpuscule (Paty, 2003). Cette période voit la naissance de la mécanique quantique. Le signe linguistique est donc, pour Saussure, une entité psychique à deux faces, comme en gros, l'onde ou le corpuscule (Mahmoudian, 2013). Saussure a bien reconnu la difficulté à conceptualiser cette consubstantialité dans une manière de penser disjonctive qui est celle de la démarche scientifique classique. De la même manière, cette consubstantialité questionne les logiques disjonctives (Bitbol, 2008) et du tiers exclu ${ }^{4}$ (Nicolescu, 2012) auxquelles nous sommes habituées depuis Aristote.

Mais si nous acceptons cette intuition partagée par Husserl et Saussure, la consubstantialité du signifiant et du signifié exprime une simultanéité de présence du signifiant et du signifié (ou de la donation de sens car celle-ci s'effectue dans la saisie conjointe du signifiant et du signifié). Le processus sémiotique ne peut commencer par une extraction de caractéristiques élémentaires du signifiant, suivi du renvoi à un signifié qui y serait préalablement associé en parcourant les différentes étapes du lexique. Il ne peut donc être pensé comme séquentiel mais doit être pensé comme un tout survenant dans un flux de conscience.

\section{II.5 - Rappels sur la théorie Saussurienne de la langue}

Il nous faut ici brièvement rappeler, de manière simplifiée, quelques éléments supplémentaires de la théorie saussurienne (pour une présentation plus détaillée voir Piotrowski, 2009 et 2013).

Pour Saussure, le continuum des sons constitue une masse amorphe (inorganisée) appelée matière ou substance de l'expression (phonèmes ${ }^{5}$ ) dans laquelle se constituent les signifiants. En regard de cette substance de l'expression existe une substance du contenu (concepts) correspondant à la masse amorphe des idées qui sera le lieu où seront institués les signifiés. Le système linguistique «plonge » à la fois dans la substance du contenu et dans celle de l'expression (figure 1). Notons que rien ne préexiste ni dans la substance du contenu, ni dans celle de l'expression.

\footnotetext{
${ }^{4}$ Le principe de bivalence rend les deux valeurs de vérité $(\mathrm{V}, \mathrm{F})$ conjointement exhaustives : « Quelle que soit la proposition p, p est soit vraie, soit fausse ». Par le principe de non contradiction: « Les deux valeurs de vérité (V, F) sont mutuellement exclusives». En vertu du principe de bivalence, la disjonction d'une proposition et de sa négation couvre exhaustivement le champ de vérité. En vertu du principe de non contradiction, la conjonction d'une proposition et de sa négation exclut la vérité. Le principe du tiers exclu postule que de deux propositions contradictoires, l'une est vraie, et l'autre fausse, ce qui exclut tout milieu, toute tierce (troisième possibilité).

${ }^{5}$ On distingue donc l'identité phonétique, à savoir la description de la matière sonore que donne la science acoustique, et qui est l'objet de la phonétique, de l'identité phonématique (ou phonémique), qui est de nature spécifiquement sémiolinguistique. Le système de la langue institue au sein d'une matière d'expression (le substrat acoustique), des identités phonématiques.
} 


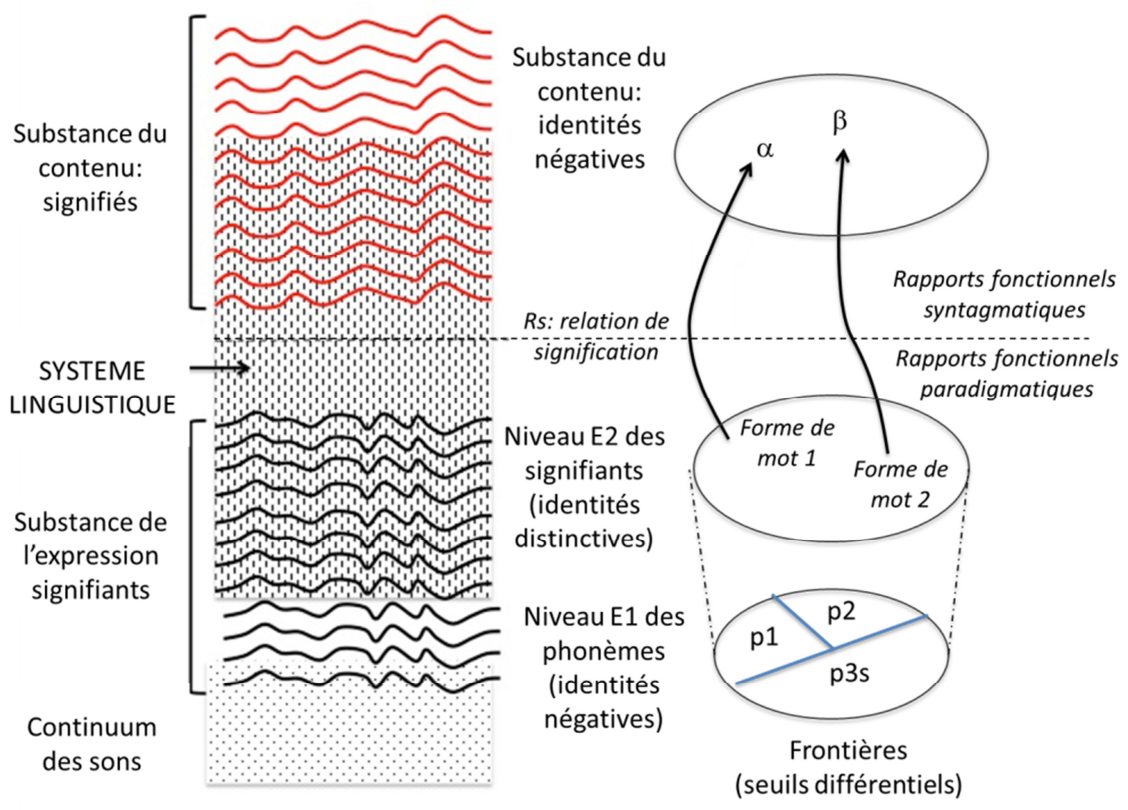

Figure 1

Le système linguistique Saussurien. Les substances de l'expression comme celle du contenu sont amorphes parce qu'inorganisées (ce caractère amorphe est symbolisé par des ondulations correspondant aux masses ondoyantes, au "royaume flottant" et aux «idées confuses » selon Saussure). Rien ne pré-existe ni dans la substance de l'expression, ni dans celle du contenu. La substance de l'expression comprend deux niveaux, E1 et E2. Dans les identités distinctives, la différence procède de la comparaison des identités ; l'identité est première sur la différence. Dans les identités négatives, on a une limitation réciproques : «chat » n'est pas «chien ».

Dans la substance de l'expression Saussure décrit un premier niveau E1, qui est celui des phonèmes ${ }^{6}$. À un niveau supérieur E2, se trouvent les signifiants ${ }^{7}$. Au plan du contenu, de même qu'au plan phonématique, les frontières que le système linguistique établit dans la substance du contenu instituent des identités oppositives. Les signifiés y sont des identités négatives construites sur des rapports de limitation réciproque : «chat » est tout ce que « chien » n'est pas.

\footnotetext{
${ }^{6}$ Les phonèmes sont les plus petites unités discrètes qui permettent de distinguer les mots les uns des autres c'est-à-dire un changement de sens (unités distinctives). L'identité du phonème $\mathrm{p}_{1}$ (ba) est précisément tout ce que le phonème $\mathrm{p}_{2}(\mathrm{pa})$ n'est pas. Les phonèmes sont ainsi des identités définies négativement qui procèdent de la catégorisation de la substance de l'expression par un réseau de seuils différenciateurs (ou frontières) y instituant des rapports de limitation réciproque.

${ }^{7}$ Les signifiants sont construits sur les phonèmes, et ils participent de rapports de différence non pas négative mais «distinctive» au sens où leurs différences résultent de leurs comparaisons. Ainsi, «chat » diffère de «chien» «[...] par le nombre, la qualité et l'ordre des unités irréductibles (Godel, 1957, p. 199).
} 
Entre les unités de la substance du contenu (concepts) et celles de la substance de l'expression (phonèmes) se trouve établie une connexion dite de «signification» (Niveau E2). La relation de «signification» (Rs) est une connexion établie non pas entre signifiant et signifié, grandeurs proprement linguistiques, mais entre occurrences concrètes de phonie et de pensée. Dans le système de la langue, la connexion conventionnelle entre chaîne phonématique et concept se trouve alors transmuée en incorporation signifiant/signifié.

Alors que les signifiés participent de rapports de différence négative, et les signifiants de rapport de différence distinctive, les signes comme totalités signifiant/signifié engagent des «oppositions ». Ce dernier mode relationnel, l'opposition, étant défini par Saussure comme une «différence conjointe à un rapport ». Sachant qu'il existe deux sortes de rapports : le paradigmatique et le syntagmatique : «[...] le jeu des différences se manifeste dans deux ordres de rapports : celui des syntagmes et celui des associations »(Godel, 1957). Ainsi, les rapports fonctionnels entre unités-signes sont de type paradigmatique (de substitution : le chat - le chien) ou syntagmatique (de juxtaposition : le chat et le chien).

\section{III - LE SIGNE SELON HUSSERL : LE LIEN AVEC LA CONSCIENCE}

Le plus souvent, dans la philosophie cognitive, il est postulé un sujet séparé d'un objet, lequel est représenté dans le sujet (conception représentationnelle). L'intentionnalité (représentationnelle) est au centre de la vie mentale et la conscience a été longtemps considérée comme secondaire ou épiphénoménale (Dupuy, 2000 ; Vion-Dury, 2007).

En revanche, dans la phénoménologie husserlienne, la conscience possède une structure intentionnelle: «toute conscience est conscience de quelque chose ». «La conscience est constituée par quelque chose qu'elle n'est pas, mais qu'elle vise à connaître car elle en est partie prenante... » (Depraz, 2002, p. 72). L'acte de conscience est décrit comme une corrélation entre un vécu intentionnel (la noèse) et un objet visé par la conscience, l'objet intentionnel (le noème). En philosophie phénoménologique, la conscience (de nature intentionnelle) est au centre de la vie mentale, et non la représentation.

Ainsi un objet n'existe dans la conscience que par la corrélation noéticonoématique qui, en quelque sorte, constitue une polarisation de la conscience vers l'objet. Ce qui n'est pas éclairé par la conscience (dans la corrélation néotico-noématique) par «le regard qui rayonne du moi pur [et] se dirige sur l'objet de ce corrélat de conscience » (Husserl, 1985, p. 284) soit n'est pas présent pour le sujet, soit apparaît dans un arrière-plan, « champ de perceptions potentielles ». Ce qui est engagé ici n'est pas en fait une problématique représentationnelle mais une problématique de type présence/absence ${ }^{8}$.

Ainsi, en ce qui concerne le signe signitif (cf $\S$ II.3), il n'y aura pas d'objet de signification « en chair et en os », là concrètement, investi par et connu dans

\footnotetext{
${ }^{8}$ On peut employer, pour illustrer ce point, la métaphore d'un sujet marchant dans une grotte obscure avec pour seule lumière sa lampe de poche. Dans le faisceau de sa lampe il peut voir une stalactite, comme lors de la noèse la conscience se dirige vers le noème. En revanche la pierre qui le fait trébucher, et que le faisceau de la lampe n'éclairait pas, n'existait pas pour lui (problématique présence/absence).
} 
cette conscience. L'objet de signification est un objet noématique, et, dans les actes signitifs ou symboliques, les actes de conscience visent ainsi un objet à vide. Le «sens » n'est pas un objet «réel» mais un objet «intentionnel » : c'est un objet « simplement visé » par la conscience : il n'a d'autre existence que «présumée », ceci à la différence des objets « réels » qui ont le caractère de l'«actuel» et de l'«effectif». La connaissance obtenue n'est pas une connaissance empirique, mais formelle.

Très précisément, dans le signe signitif, l'intentionnalité signitive se saisit d'une matière phonique (objet primaire) et, simultanément, la polarise en tant que visant un sens (signifié) pour la rendre comme expression: le signifiant. C'est ainsi que se produit dans la conscience l'unité de la manifestation « consubstantielle » du signifiant et du signifié.

\section{III.2 - La conscience verbale}

La «conscience verbale » afférente au "phénomène signe» coordonne organiquement, suivant les modalisations d'un champ attentionnel, les visées respectives d'une intentionnalité perceptive et signitive, qui se trouvent ainsi promues, respectivement, au rang de signifiant et de signifié. La conscience est une structure globale qui est le lieu de modalisations sous l'effet de la modulation par le champ attentionnel. Si nous devons assumer la coprésence du signifiant et du signifié dans ce cadre, il nous faut envisager la conscience également comme un champ ${ }^{9}$ dans lequel se donnent en même temps le signifiant et le signifié. Une métaphore simple et musicale permet d'approcher cette simultanéité. Dans un accord de do mineur plaqué, les quatre notes (do, mi $b$, sol, do) sont coprésentes et l'audition de chaque note détermine l'audition de toutes les autres: si sur la partition elles apparaissent artificiellement stratifiées, dans l'audition, elles n'apparaissent pas séquentielles, mais simultanées. Il nous faut faire un effort de décomposition, secondaire et d'ordre réflexif, pour y retrouver la tonique, la fondamentale et la tierce mineure. Toutes les notes sont consubstantielles à l'accord. Par comparaison, un dispositif de type fonctionnaliste de la langue se comprendrait plutôt comme un accord arpégé, dans lequel on entendrait la séquence des notes séparées par des silences; ce dispositif laisse d'ailleurs la porte ouverte, dans le cas où les notes de l'accord arpégé seraient liées, à une rétro-action de l'effet des notes l'une sur l'autre mais le type de co-présence n'est évidemment pas le même que dans l'accord plaqué.

Dans les modèles séquentiels, on a implicitement une base de temps (en abscisse) et l'accès lexical se fait en parcourant, selon cette base de temps tous les modules nécessaires. Dans ce cas, le présent est pensé comme un point sur l'axe des temps. Il n'en n'est pas de même de la temporalité qui accompagne toute expérience vécue et se déploie dans divers horizons de durée : coexistent le présent de l'impression et ses vécus constitutifs plongeant dans les horizons du passé (rétention) et du futur (protension) (Husserl, 1985, p. 277). C'est ce

\footnotetext{
${ }^{9}$ « Ne pourrait-on pas appeler de même champ de la conscience ou étendue maximum de la conscience, le nombre le plus grand de phénomènes simples ou relativement simples qui peuvent se présenter à la fois dans une même conscience [...] ? » (Janet, 2005, p. 148).
} 
que l'on nomme le présent «spécieux $»^{10}$ (Depraz, et $a l, 2011$, p. 177). Ce présent, on le voit, n'a pas l'aspect d'un point sur une droite: une consubstantialité du signifiant et du signifié peut en quelque sorte s'y loger.

Enfin, il nous faut considérer la conscience non pas comme la seule conscience réflexive, consciente d'elle-même mais comme le tout formé de la conscience réflexive («consciousness ») et de la conscience non-réflexive («awareness », Husserl, 1985). Une grande partie des processus que nous décrivons se tient dans cette dernière et ne nous sont pas immédiatement accessibles par la réflexion et dans la conscience réflexive. Dès lors, dans une conscience réflexive, les processus nous apparaissent volontiers comme séquentiels alors qu'ils sont largement co-présents dans une conscience préreflexive (Vermersch, 2012) ${ }^{11}$.

Pour Husserl, le «phénomène signe » se distribue sur quatre strates de conscience verbale ${ }^{12}$ toutes coprésentes dans le champ de conscience (Husserl, 2003) :

- a) la conscience de son de mot: on entend «pomme » en tant que son concret « pom » fermé sur lui-même. C'est ce caractère «fermé sur lui-même » qui caractérise phénoménologiquement le «son de mot » husserlien. Ainsi, au plan de la conscience verbale, Husserl ne fait pas de distinction entre le phonétique et le phonémique ${ }^{13}$.

- b) la conscience primaire (ou, dans le vocabulaire saussurien, conscience de signifiant) : «pomme ». C'est une conscience d'objet (attentionnel) primaire, c'est-à-dire d'un objet en tant que «tourné » vers une certaine signification vers laquelle il oriente la conscience comme à sa pleine existence.

- c) la conscience (attentionnelle) «thématique »: elle concerne l'objet de signification que la conscience investit pleinement au sens où, suivant les termes de Husserl, elle y «habite ». Dans une perspective

\footnotetext{
${ }^{10}$ La notion de présent spécieux vient de James, qui écrit : « Nous connaissons si peu la nature intime de l'activité cérébrale que, même quand une sensation persiste de façon monotone, nous ne pouvons pas dire que ses premiers moments ne laissent pas des processus d'évanouissement qui coexistent avec ceux du moment présent. La durée et les évènements forment tout ensemble notre intuition du présent spécieux [specious] avec son contenu ». (James, 2003, pp. 633-634). On voit que spécieux veut dire ici trompeur, ambigu. En même temps, on peut interpréter et décrire ce présent spécieux comme épais car contenant les protensions et les rétentions husserliennes.

${ }^{11}$ Outre la métaphore d'accord, on peut penser, à partir de la description husserlienne légèrement complétée, la conscience verbale comme un vecteur d'état de la mécanique quantique comprenant plusieurs états pondérés par un coefficient, coprésents et liés, mais différenciables par des processus expérientiels ou expérimentaux.

${ }^{12}$ Ces strates correspondent à des modalisations de la conscience verbale, le terme modalisation luimême utilisé par Husserl permettant de mieux comprendre l'organisation de cette conscience verbale.

${ }^{13}$ La position de Husserl est différente de celle de Jakobson qui attribue au phonème un «signifié zéro », et lui reconnait donc une ouverture vers le sens. Husserl (ainsi que Hjelmslev) dénie aux phonèmes et aux logatomes toute épaisseur signifiante. Pour Husserl, la seule distinction pertinente concerne l'existence ou non d'un «authentique » signifié porté par le signifiant. Ainsi les phonèmes comme les logatomes relèvent d'une simple conscience de «son de mot» et non sont pas reconnus comme de vrais signifiants qui, quant à eux, relèvent d'une conscience «primaire » tournée vers un signifié.
} 
structurale, il s'agit du signifié comme identité différentielle de sens institué dans la substance de contenu : à savoir «pomme» en tant qu'opposé à « poire »; et enfin :

- d) la conscience de remplissement, qui est l'acte par lequel on passe d'un objet simplement intentionné (présomption d'objet) à un objet actuel (une image mentale).

À la source de ce processus se produit ce que l'on pourrait appeler une polarisation de la matière phonique.

Dans le champ de la conscience verbale, l'objet primaire (signifiant) oriente vers (et coexiste avec) l'objet thématique (signifié) et les quatre composantes de ce champ de conscience sont simultanément présentes.

\section{IV - LA SYNTHÈSE ENTRE SAUSSURE ET HUSSERL : QUE MANQUE-T-IL ? LES MODÈLES MORPHODYNAMIQUES}

\section{IV.1 - Un premier bilan}

Nous avons vu que dans les propositions saussuriennes et husserliennes, le signifiant est reconnu consubstantiel au signifié (Saussure) et l'accès au sens est décrit comme s'effectuant dans un acte de conscience intentionnel qui « polarise » l'objet de conscience simplement perceptif, le son de mot, en objet de conscience primaire (le signifiant), donc comme objet orienté vers un objet thématique (signifié) dans une corrélation noético-noématique (Husserl, 1985, pp. 300-332). Ici, nul clivage entre signifiant et signifié.

Ces deux perspectives théoriques sont complémentaires. Mais il manque, pour réunir ces deux dispositifs théoriques un dispositif formel résolument non séquentiel, accomplissant l'assimilation de la théorie structuraliste à celle de la conscience verbale.

\section{IV.2 - Les modèles morphodynamiques du signe : une solution au problème de la consubstantialité}

La solution proposée ici est d'élaborer une théorie morphodynamique du signe intégrant les processus de création permanente, au cours du discours, d'unités différentielles de sens (signifiés) par l'émergence de frontières; dans un continuum sémantique (processus de catégorisation). Il s'agit d'une théorie organisatrice de la forme (morphogenèse) qui ne reconnait pas le caractère séquentiel de production des entités pour lier toute variation d'une entité à la variation simultanée de toutes les autres.

Le modèle morphodynamique, est tout d'abord un modèle dynamique et s'intègre dans l'émergence de la conception des systèmes neurocognitifs comme des systèmes dynamiques plutôt que computationnels (Bassett \& Gazzaniga, 2011). Or en physique, les systèmes dynamiques fonctionnent sans représentation, sans computation, sans opérations séquentielles ou cycliques, sans symboles. Le fonctionnement du système entier est uniforme et non décomposable ; tout y est contraint par le temps. Le changement d'un seul paramètre parmi tous les autres modifie la dynamique totale et affecte en temps réel la valeur de toutes les autres variables du système. 
Le modèle morphodynamique (Thom, 1977; Petitot-Cocorda, 1985; Bompard-Portc \&Thom, 1994 ; Pctitot-Cocorda, 1997 ; Pctitot-Cocorda, 2009) relève comme son nom l'indique d'une théorie générale de la forme. Il décrit les conditions d'émergence et de variation de formes. Le modèle morphodynamique est approprié à une mathématisation du structuralisme saussurien. Il permet de décrire et d'expliquer le découpage d'un continuum (c'est-à-dire en fait la catégorisation) et la covariation (orientée en termes d'un " contrôle ") dans la substance du contenu ou dans la substance de l'expression (configurée en chaînes phonématiques), conduisant respectivement aux signifiants et aux signifiés. On montre par ailleurs que l'approche morpho-dynamique du signe produit une analyse de l'unité indivise signifiant-signifié (Piotrowski, 2009). A la différence du paradigme logico-computationnel qui rapporte les mécanismes mentaux à des traitements d'unités symboliques, la modélisation morphodynamique approche donc les processus cognitifs en termes d'interaction et de stabilisation de systèmes dynamiques.

\section{IV.3 - Caractéristiques générales des modèles morphodynamiques}

Le modèle morphodynamique du signe, propre à réunir coappartenance forme-sens, système dynamique, morphogenèse, est également pourvu d'une signification phénoménologique, en termes de modalisation de la conscience verbale (voir Piotrowski, 1997 ; Piotrowski, 2009, p. 307 ssq. ; Petitot, 2008 pour plus de détails sur ce modèle). Nous disposons ainsi d'un modèle explicite articulant et synthétisant les vues phénoménologiques et structuralistes, et rendant justice autant conceptuellement qu'architecturalement à l'unité indivise du signe.

Dans le modèle morphodynamique (figure 2), les dynamiques sont définies en termes d'attracteurs (objets mathématiques spécifiant un état vers lequel un système peut converger) qui déterminent des états en concurrence pour l'actualisation: l'état actualisé est celui qui correspond à l'attracteur en position de minimum absolu, les états relatifs aux autres attracteurs (minimum relatifs) sont alors rendus virtuels, mais restent présent en termes d'une compétition à l'actualisation, en quelque sorte à l'arrière-plan (cf. § III.1).

Dans le modèle standard de la mécanique, les dynamiques, dont les attracteurs déterminent les états stables possibles, sont qualifiées de manière extrinsèque, sur la base d'un repère prédéterminé. Dans le modèle morphodynamique les dynamiques ne sont pas déterminées relativement à un repère externe : c'est leur type qualitatif (nombre et positions relatives des attracteurs), qui en est une propriété "intrinsèque » (i.e. indépendante des repères), qui se trouve seul pris en considération. Pour certaines variations du paramètre de contrôle, on observe un changement brutal («catastrophique" (Thom, 1977)) et le type qualitatif de la dynamique contrôlée : l'état actuel (minimum absolu), en compétition à des états virtuels, devient alors virtuel au profit d'un des autres états qui s'actualise. On catégorise ainsi l'espace de contrôle en domaines délimités par des frontières dont les valeurs déterminent 
À tout élément $A, B, H$ de

$W$ est associé un élément

$f_{A}, f_{B}, f_{H}$, dans $F$.

$f$ est une fonction

En H: dynamique instable instituant la frontière $\mathrm{Kw}$

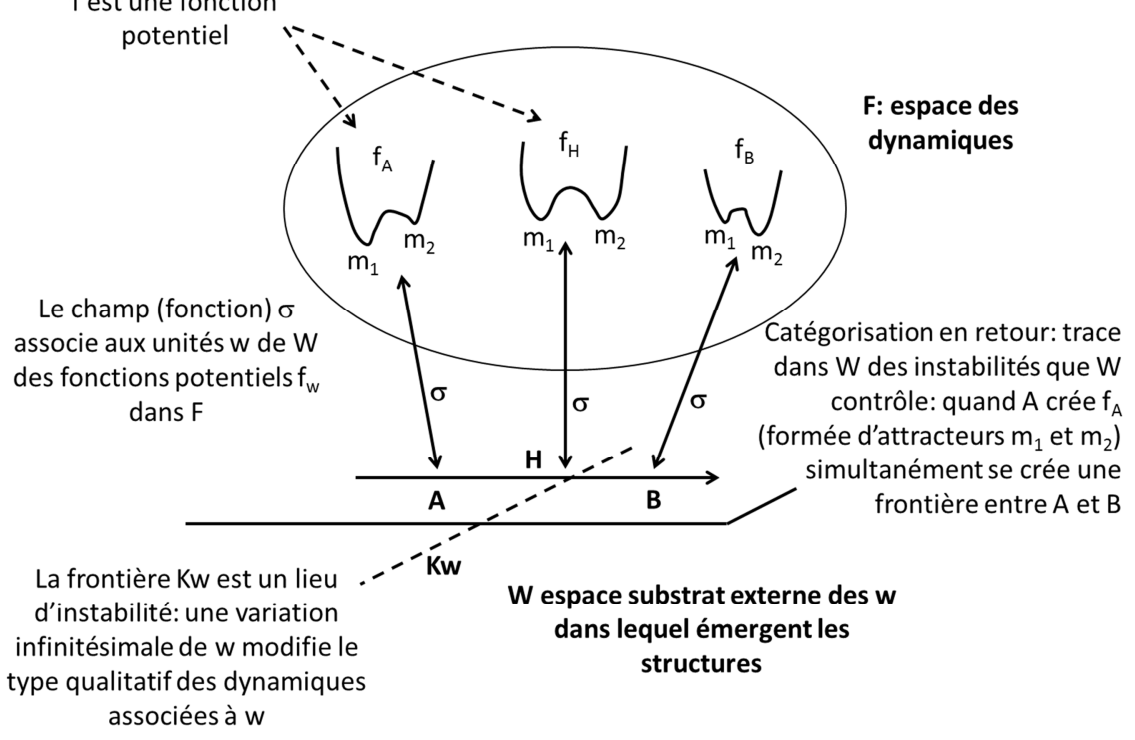

Le modèle morphodynamique. Ce modèle fait intervenir deux espaces : un espace externe, $\mathrm{W}$ et un espace de dynamiques, $\mathrm{F}$. L'espace $\mathrm{W}$ est un espace substrat homogène et au sein duquel il s'agit d'expliquer la formation de discontinuités catégorisantes (système de seuils différenciateurs); il ne détient pas en propre le principe de sa catégorisation. On le lui adjoindra en le «plongeant» dans un espace de complexité supérieure, l'espace $\mathrm{F}$ de dynamiques (caractérisées comme fonctions potentiel à valeurs réelles), suivant le mode d'un champ de contrôle $\sigma$ (champ de $\mathrm{W} \rightarrow \mathrm{F}$ ) ; en retour, W héritera des hétérogénéités de F. Le champ $\sigma$ associe aux unités $w$ de $\mathrm{W}$ des fonctions potentiel $\mathrm{f}_{\mathrm{w}}$ de $\mathrm{F}$. Par convention, l'état « actuel » du système correspond au minimum absolu de $\mathrm{f}_{\mathrm{w}}$, les états correspondant aux autres minima (relatifs) étant alors «virtuels». Ici, les éléments A, B, H de W déterminent les dynamiques $\mathrm{f}_{\mathrm{A}}, \mathrm{f}_{\mathrm{B}}, \mathrm{f}_{\mathrm{H}}$ qui présentent chacune deux minima $\mathrm{m}_{1}$ et $\mathrm{m}_{2}$ (attracteurs en compétition pour l'actualisation) positionnés de différentes façons (minima absolu, relatif ou égaux). Les dynamiques $\mathrm{f}_{\mathrm{A}}$ et $\mathrm{f}_{\mathrm{B}}$ sont «stables», au sens ou une modification infinitésimale de leur «profil» n'en modifie pas le type qualitatif: les attracteurs $\mathrm{m}_{1}$ et $\mathrm{m}_{2}$ conservent leurs qualifications respectives de minima absolu ou relatif. A l'inverse la dynamique $\mathrm{f}_{\mathrm{H}}$ est instable car une altération infinitésimale la transforme en dynamique du type de $\mathrm{f}_{\mathrm{A}}$ ou de $\mathrm{f}_{\mathrm{B}}$. La dynamique $\mathrm{f}_{\mathrm{H}}$ associée à $\mathrm{H}$ constitue donc une singularité qui catégorise le continuum $\mathrm{W}$ : les configurations concurrentielles (entre attracteurs) que les dynamiques déterminent restent inchangées de A à $\mathrm{H}$ (exclu), puis en $\mathrm{H}$ la dynamique associée présente un type qualitatif instable qui, en retour, institue $\mathrm{H}$ en qualité de frontière Kw séparant deux types de configurations dynamiques, et passé cette frontière, de $\mathrm{H}$ (exclu) à $\mathrm{B}$, les valeurs de $\mathrm{W}$ déterminent de nouveau des dynamiques qualitativement identiques. Ainsi la «traversée » de Kw (ensemble des points singuliers) suivant un chemin (générique) de W se traduit par une modification « catastrophiste » (Thom, 1977) (i.e. fait «basculer» d'un type de forme à un autre type de forme) des dynamiques associées. Il s'agit d'une modification non des valeurs des attracteurs des fonctions potentiel mais des formes des dynamiques (identités qualitatives) de F. 
des systèmes instables, et de part et d'autre desquelles ces instabilités se stabilisent en dynamiques de types qualitatifs distincts

Il convient encore d'insister sur le fait que toute variation dans l'espace $\mathrm{W}$ (espace externe dans lequel émergent les structures) sera associée à une variation dans l'espace $\mathrm{F}$ des dynamiques et réciproquement, par la vertu même d'un système dynamique.

\section{IV.4 - Application des modèles morphodynamiques à la linguistique saussurienne}

Appliqué à la théorie saussurienne du signe, le modèle rend compte de la constitution d'identités différentielles de sens au sein d'une substance de contenu supposée homogène (la «masse amorphe des idées »). L'espace F des dynamiques correspond en gros au système cognitif considéré - ce qui fut le cas pour les théories modularistes - comme une boîte noire. La substance du contenu est l'ensemble de tous les sens possibles actualisés ${ }^{14}$.

Les identités sémantiques considérées sont des valeurs négatives et oppositives (à savoir des signifiés) : «pomme » est tout ce que «poire » n'est pas. Ainsi, du côté des A, on aura par exemple tous les sens actualisés de «pomme» et du côté des B, tous les sens actualisés de «poire». Dans le modèle PMS, ces «négativités » sont rendues par un réseau de frontières dans l'espace W (l'espace substrat externe ou substance du contenu - dans lequel émergent les structures); il s'agit d'une catégorisation différentielle. Les «oppositivités » sémantiques sont, elles, rendues en termes de compétitions à l'actualisation des attracteurs de $\mathrm{F}$ portés par les dynamiques que les occurrences de $\mathrm{W}$ déterminent.

La fonction de contrôle $\sigma$ relie donc des éléments $w$ de la substance du contenu (tous les sens actualisés) à des configurations dynamiques dans le système cognitif $(\mathrm{F})$ considérées du point de vue de leurs types qualitatifs. La catégorisation linguistique s'effectue alors en retour entre les types de configurations dynamiques du système cognitif et la substance du contenu. Ainsi à toutes les significations actuelles de "pomme » (rouge ou verte) on associera (par $\sigma$ ) des dynamiques cognitives (des types d'attracteurs) similaires en tant qu'elles s'opposent à «poire», et en retour à toute pomme (par exemple jaune), correspondra une forme de mot «pomme» distincte du mot «poire». De même pour toutes les «poires », de l'espèce «doyenné des comices » à la «williams», les configurations (les types dynamiques) du système cognitif seront identiques.

On saisit tout autant que «poime» ou «pore » possède un sens instable (c'est un point $\mathrm{H}$ sur la frontière $\mathrm{K}$ ), qui génère dans le système cognitif une configuration ambiguë («poire», «pomme»?, $f_{H}$ ) que l'on a envie de résoudre (figure 3)

\footnotetext{
${ }^{14}$ C'est-à-dire tous les sens possibles qu'a pris un mot comme le mot pomme dans l'expérience d'un individu : pomme rouge, golden, la pomme de la marque « apple», etc.
} 


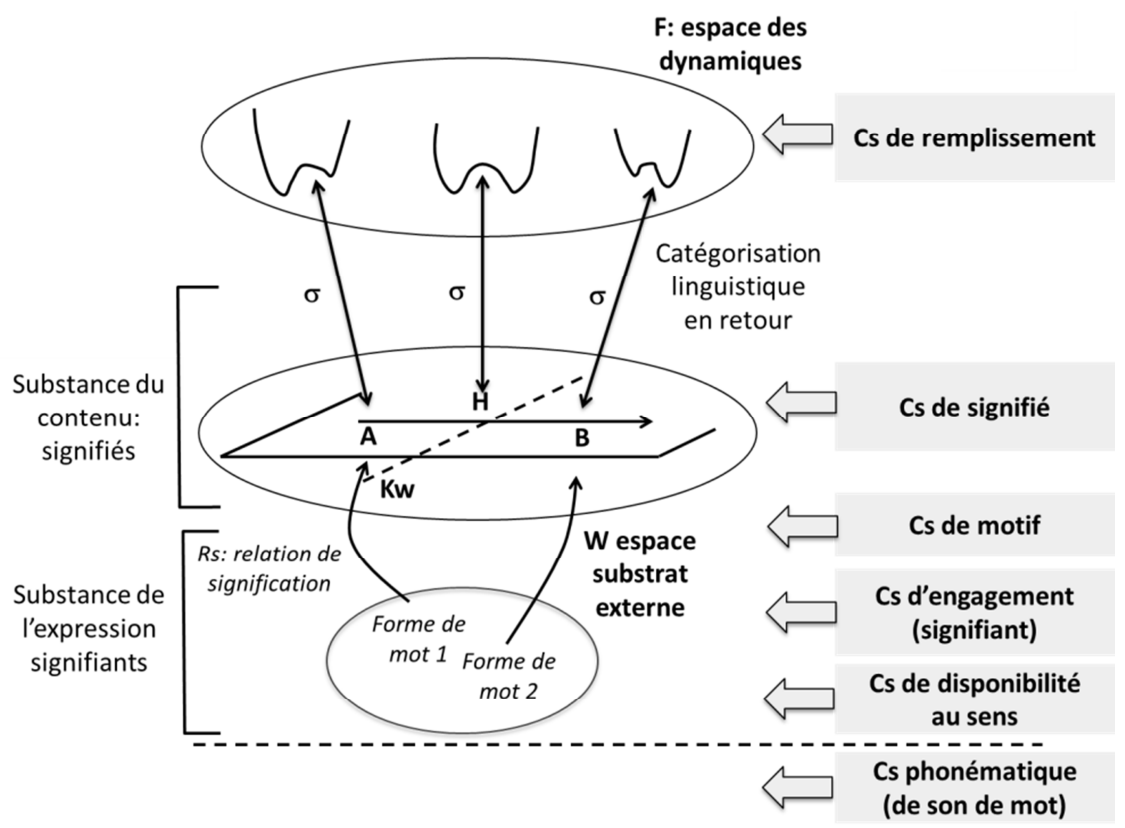

Figure 3

Application du modèle morphodynamique à la linguistique Saussurienne. Ce schéma est la synthèse simplifiée des figures 2 et 3 . Les modalités de conscience (superposées dans la figure) doivent être comprises comme les notes d'un accord plaquées : simultanées, consubstantielles mais artificiellement décomposables. (voir IV.2)

La composition des relations de signification (reliant des formes de mots à des éléments de la substance du contenu ${ }^{15}$ ) avec le champ $\sigma$ met en connexion fonctionnelle les formes de mots avec l'espace des dynamiques (F). Le schéma fonctionnel morphodynamique permet de tenir ensemble signifiant et signifié dans un rapport de consubstantialité en raison du fait que l'ensemble du système covarie.

\footnotetext{
${ }^{15}$ Hjelmslev porte un regard critique sur la différenciation de Saussure entre les substances de l'expression et de contenu. Pour Hjelmslev, la langue ne se constitue pas «entre » deux masses amorphes mais dans les rapports de dépendance établis entre la forme de l'expression et celle de contenu. Hjelmslev distribue hiérarchiquement deux sortes d'articulation: entre forme et substance d'une part, et entre «plans » d'expression et de contenu d'autre part, la seconde articulation précédant hiérarchiquement la première. On aura donc une forme et une substance de l'expression, et une forme et une substance du contenu (Hjelmslev, $1971 ; 2000)$. Ainsi en tenant compte de cette nuance entre forme et substance le modèle que nous proposons se rapproche également de la pensée de Hjelmslev.
} 


\section{V - LIENS ENTRE LA THÉORIE MORPHODYNAMIQUE DU SIGNE ET LES DIFFÉRENTES ASPECTS (MODALITÉS, ÉTATS) DE CONSCIENCE VERBALE : LE MODÈLE PHÉNOMÉNOLOGIQUE ET STRUCTURAL DU SIGNE}

Disposant désormais des concepts nécessaires de la linguistique saussurienne, de la conscience verbale husserlienne et des approches topologiques de la morphodynamique, il nous est désormais possible de relier l'ensemble pour une description plus synthétique du modèle proposé. En effet, le modèle morphodynamique qui rend formellement compte de la théorie saussurienne du signe par une topologie des relations dynamiques (non séquentielles mais simultanées et co-occurrentes) débouche sur une lecture phénoménologique qui lie le modèle saussurien au dispositif husserlien des modalisations de la conscience verbale.

Il est important de souligner de nouveau le caractère holiste de l'architecture morphodynamique : cette architecture est à comprendre comme une grille systémique unitaire qui, dans un seul temps opératoire, se saisit d'un matériau empirique (les sons de mots et leurs liens de signification) et, sous une visée globale de constitution de sens, l'informe comme complexe sémiotique modalisé. Dans ce complexe, signifiants et signifiés se déterminent réciproquement.

Il reste qu'une telle architecture fonctionnelle peut être approchée, pédagogiquement, en modalités (ou modalisations), en portant successivement au premier plan chacune des composantes qui participent d'une conscience verbale intégrée. Il ne s'agit pas de démembrer le dispositif morphodynamique en une succession de plans d'objets, mais on peut y distinguer sur le mode d'une «mise en relief » différentes modalisations qui, dans des logiques de recouvrement réciproques, participent de la texture du signe dans son déroulé naturel vers le sens et ses remplissements.

Chacune de ces modalisations procéde de la sélection et de la mise en relief de certaines composantes structurales et/ou fonctionnelles et, corrélativement, de la neutralisation ou du «passage en arrière-plan » de celles qui ne sont pas retenues.

Cependant, le dispositif Husserlien reste relativement grossier car il pense une distribution avec seulement des places pour le signifiant et le signifié (plus le remplissement). Il ne permet pas de penser notamment la problématique du pseudomot (comme «pradesutima»). Il faut donc compléter le dispositif husserlien; c'est ce que fait le modèle morphodynamique qui, en raison de son organisation topologique, présente une granularité plus fine que l'analyse husserlienne. Il permet des articulations et des modalisations de la conscience verbale intermédiaires ou subdivisant les plans retenus par Husserl.

Ainsi, aux quatre modalisations de la conscience verbale décrites par Husserl (conscience de son de mot, conscience primaire, conscience thématique, conscience de remplissement), le modèle PMS rajoute la conscience de disponibilité et la conscience de motif (figure 3).

La conscience de son de mot (évènement acoustique) ne fait pas partie de l'acte sémiotique. Les modalisations suivantes sont proprement sémiolinguistiques : leurs objets procèdent de positions systémiques partielles, puis progressivement élargies, jusqu'à solliciter la totalité du dispositif 
morphodynamique. Les deux premières modalités de conscience verbale (de disponibilité et d'engagement) détaillent ce que Husserl rapporte sous la seule conscience «primaire » de signifiant - cette conscience d'objet "primaire» étant celle d'une modalisation par laquelle une extériorité (un son de mot) se trouve internalisée à titre de signifiant. Le «son de mot» prend statut de signifiant en ce qu'il se voit attribuer une certaine position fonctionnelle : celle de tourner la conscience vers du sens.

\section{V.1 - La conscience de son de mot}

Il y a d'abord la conscience simplement perceptive de «son de mot», à savoir une conscience de perception phonématique, mais en tant que «fermée sur elle-même » (et qui en ce sens ne se distingue pas au plan de son implication sémiolinguistique d'une conscience phonétique). Mais à ce stade, déjà, la conscience fait une différence entre les non-mots (qui sont simplement des suites de phonèmes (ou de graphèmes) imprononçables type «prwsesirtsd ») et les chaînes phonématiques conformes aux exigences du système phonologique d'une langue donnée: les mots et les pseudomots (ou logatomes). Car la perception phonématique est une perception de morphologies, c'est-à-dire une perception de totalités organiques où l'identité des parties procède de l'identité du tout qui les intègre. Ainsi les mots et les pseudomots, en tant qu'ils satisfont à des exigences de bonne formation, se laissent pleinement percevoir sur un mode phonématique, et se distinguent ainsi des non-mots qui, quant à eux, font obstacle à l'accomplissement d'une perception phonématique. C'est cette morphologie phonématique (des mots ou des pseudomots) qui «sollicite» l'intentionnalité sémiolinguistique. On ne peut cependant éliminer le fait que la conscience de son de mot saisisse aussi l'aspect prosodique dans la mesure où toute prononciation naturelle présente un caractère prosodique.

\section{V.2 - La conscience de disponibilité}

La conscience verbale qui correspond à un complexe phonique en tant qu'il tombe sous un acte de l'intentionnalité sémiotique, est une simple conscience de disponibilité au sens où le signifiant est saisi seulement comme susceptible de participer d'une configuration verbale à venir. La conscience de disponibilité au sens n'est rien d'autre qu'une conscience de ce moment critique de «basculement vers...», sans détermination aucune de l'orientation ou même de la possibilité d'accomplir effectivement un tel basculement.

\section{V.3 - La conscience d'engagement}

À un degré d'élaboration supérieur, et «au contact» de la conscience de disponibilité, sera engagée la connexion fonctionnelle de contrôle, mais du seul point de vue de son existence (abstraction faite de son identité propre, à savoir sa connexion à telle ou telle région de contenu). L'objet de conscience ainsi retenu procède d'une simple conscience d'engagement (au sens). C'est le plan de la «signifiance sémiotique »(Benveniste, 1973), plan sollicité a minima dans les épreuves de «décision lexicale » où il s'agit de reconnaître un item dans sa qualité de mot ou de pseudomot, les autres modalisations de conscience verbale pouvant de même servir d'appui pour la décision lexicale). 


\section{V.4 - La conscience de motif}

La conscience de motif (Cadiot \& Visetti, 2001) est la modalisation qui sollicite la connexion fonctionnelle à la substance du contenu dans son identité spécifique (orientation particulière) mais sans qu'une conscience de signifié, qui relève de la modalité ultérieure, ne soit encore sollicitée. Le concept de « motif » réhabilite V.4Vl'unité sémantique du morphème.

La conscience de motif procéde de l'identité («motif») de la connexion fonctionnelle entre les unités d'expression et la substance de contenu: elle nourrit, au travers des schémas de différentiation dynamique du modèle morphodynamique, la conscience du signifié. La conscience de «motif» correspond à la possibilité d'une conscience de sens qui ne soit pas encore une conscience de signifié. Comme conscience de sens, la conscience de motif rapporte «[...] une dynamique de constitution d'un rapport à..., d'un accès vers..., d'un mode d'appréhension, de donation, de construction... » (Cadiot \& Visetti, 2001, p. 114).

La conscience de motif, au contraire de la conscience de signifié, n'ouvre pas la possibilité d'effectuer des tests de catégorisation sémantique.

\section{V.5 - La conscience de signifié}

La conscience de signifié, qui chez Husserl correspond à la «conscience thématique » (conscience de l'objet de signification), est précisément la conscience d'un sens différentiel. Cet aspect de la conscience «capte » un contenu en tant qu'il s'oppose à un autre contenu ( « poire »). C'est donc une conscience de limitations réciproques qui, dans le modèle morphodynamique, sont rendues et articulées par la frontière $\mathrm{K}$.

\section{V.6 - La conscience de remplissement}

La dernière modalité de la conscience verbale est la conscience de remplissement, non explicitement située dans le schéma morphodynamique. Il s'agit dans l'acte du remplissement de porter un objet négatif et simplement intentionnel (le signifié) à un degré de positivité et d'effectivité supérieur, à travers par exemple, l'actualisation d'une représentation ${ }^{16}$ mentale, ou une détermination catégorielle, ou encore le renvoi à un référent. On sort ici du champ sémiolinguistique. Dans le modèle morphodynamique, c'est la conscience de telle ou telle occurrence possible du mot «poire » (Williams, Comice, Abate, etc.) dans la substance du contenu.

\section{VI - PARTICULARITÉS DU MODÈLE MORPHODYNAMIQUE DANS LE «PAYSAGE LINGUISTIQUE »}

\section{VI.1 - Linguistique et sciences cognitives}

Avant d'envisager la mise à l'épreuve du modèle PMS par la neurophysiologie, il nous faut positionner succinctement celui-ci dans le

${ }^{16}$ Ici le mot représentation est pensé dans une acception phénoménologique et non cognitiviste. 
«paysage» de la linguistique contemporaine. L'exercice est délicat pour plusieurs raisons : 1) il existe un nombre important de propositions explicatives et de modèles en linguistique ; 2) à ces modèles linguistiques se superposent des modèles de neurosciences cognitives compatibles ou incompatibles avec tel ou tel modèle linguistique; 3) il se produit une évolution très rapide des modèles ainsi que l'apparition de nouveaux modèles dans les deux disciplines et 4) l'implémentation éventuelle des modèles linguistiques dans un cerveau «en action» est extrêmement difficile, compte tenu des méthodes actuelles d'imagerie - au sens large - dont nous disposons. Le cadre de cet article ne nous permet pas de réaliser une revue générale de la littérature en linguistique. Cependant nous allons tenter de positionner ce modèle phénoménologique dans le champ des modèles linguistiques et neurolinguistiques proposés.

Le cognitivisme symbolique classique est a) représentationnaliste (il existe des représentations mentales implémentées dans des systèmes neurologiques) ; b) computationnaliste (il postule que les représentations traitent de l'information en activant des processus syntaxiques plus que sémantiques) et propositionnel ${ }^{17}$ (traitement de propositions logiques) et, c) il est fonctionnaliste dans la mesure où on peut découpler le niveau matériel bio-physique et le niveau logicosymbolique (Petitot, 1993). De plus les systèmes fonctionnalistes ne sont pas intentionnels. Par contraste, les propositions phénoménologiques (husserliennes) soutiennent que les idéalités corrélatives des actes mentaux ne sont pas propositionnelles et que l'intentionnalité est constitutive des représentations mentales (Petitot, 1993).

Le cognitivisme classique a été d'abord un cognitivisme modulariste et atomiste, dans la mesure où il postulait des modules théoriques de traitement de l'information, laquelle était par ailleurs scindée en «atomes» traités séquentiellement. Il est probable que ce sont les progrès des ordinateurs et des théories de l'information qui ont ensuite donné une direction différente au cognitivisme. C'est le cas notamment du réseau de neurones idéalisés de Mac Culloch et son implémentation dans le perceptron de Rosenblatt. De représentationnaliste et computationnaliste, le cognitivisme est devenu connexionniste, distribué, dynamique et a été le lieu de processus émergents (Vion-Dury, 2007; Dupuy, 2013). Cependant, selon les auteurs, soit le connexionnisme est resté représentationnaliste, soit il est devenu énactif, la représentation faisant place à des processus dynamiques et émergents (Varela, et al., 1993, p. 207 ssq.).

La linguistique contemporaine a, par le biais de la philosophie analytique, largement contribué au cognitivisme. La linguistique de Chomsky était essentiellement syntaxique, atomiste et séquentielle (Gardner, 1993, p. 212 ssq.), alors que certaines propositions actuelles s'inspirent largement du

\footnotetext{
${ }^{17}$ Les systèmes cognitifs sont largement inspirés par les philosophes de la logique et du langage (Frege, Wittgenstein, Russel, Ryle notamment) dans laquelle le langage va apparaître comme un calcul logique sur les symboles qui représentent la réalité, en insistant sur la différence entre sens et référence. On appelle «tournant linguistique » (« linguistic turn »), le moment où le langage est à l'origine d'une conception de l'esprit dans laquelle a) les pensées sont «localisées » dans le langage et, b) sur un plan philosophique, dans la proposition que l'explication philosophique de la pensée se résout dans l'explication philosophique du langage

La philosophie analytique sera un des fondements de la philosophie cognitive (Dupuy, 2013).
} 
connexionnisme et des processus dynamiques et distribués en faisant appel à des attracteurs. La linguistique a été marquée par les idées développées au début du cognitivisme et, d'une certaine manière, semble aussi en avoir suivi l'évolution.

On peut proposer une sorte de typologie des modèles linguistiques, à partir deux groupes de caractéristiques: a) distribués versus symboliques ou atomistes et $b$ ) séquentiels versus interactifs/dynamiques. On aura ainsi 4 types de modèles principaux : a) connexionnistes, qui sont distribués, interactifs et dynamiques; b) symboliques, qui sont séquentiels et modularistes; c) symboliques, qui sont interactifs et dynamiques et d) distribués et séquentiels. Notre modèle fait partie du premier type de modèles.

\section{VI.2 - Modèles linguistiques connexionnistes, dynamiques et distribués}

Nous porterons notre attention non plus sur les approches modularistes de la linguistiques mais plutôt sur les courants théoriques qui se rapprochent le plus de notre modèle, à savoir les courants dynamicistes.

Les modèles dynamiques développés en linguistique ont en commun de considérer les «états» linguistiques observables (par exemple, les configurations syntaxiques ou les valeurs sémantiques) comme résultant de processus dynamiques sous-jacents et dont ces «états » constituent donc les «attracteurs ». La dynamique qui fixe l'état vers lequel le système converge est contextuellement déterminée. Ces modèles ont aussi en commun de se donner au départ, par le choix d'un référentiel particulier, un espace d'états possibles parmi lesquels la dynamique en sélectionnera un ou un ensemble.

Il en est ainsi, par exemple, du modèle de Fuchs et Victorri où la valeur actualisée de l'adverbe « encore » est caractérisée en fonction des contextes par telle ou telle plage d'un espace de significations défini sur une base particulière (un référentiel) de traits sémantiques (Victorri \& Fuchs, 1992). Dans ces approches, et contrairement à la nôtre, les dynamiques ne déterminent donc pas des morphologies différentielles instituant des référentiels sémantiques locaux, mais des sous-ensembles de valeurs sémantiques préalablement qualifiées dans un espace global. Cela n'empêche pas ces systèmes dynamiques d'être sur le plan neurophysiologique, connexionnistes et non modularistes (Victorri \& Fuchs, 1996).

La conception dynamiciste traverse aussi l'approche énonciative de Culioli (Culioli, 1991 ; 2000). La TOE (Théorie des Opérations Énonciatives) étudie, comme dans le modèle morphodynamique, en effet, comment la langue installe, des frontières dans des espaces sémantiques et la production de valeurs sémantiques dans un espace de signification encore indéterminé (La Mantia, 2014). On retrouve bien chez Culioli une conception topologique et dynamique des structures et des opérations langagières. Parmi les concepts qu'il met en œuvre à des fins descriptives, on trouve notamment ceux d'intérieur/extérieur, de voisinage, de frontière, d'homogénéité... Le caractère dynamique est quant à lui principalement rendu par la notions d'attracteur et de bassin d'attracteur, ainsi que par les jeux de bifurcations. Mais la proposition de Culioli ne rend pas compte des principes internes d'une genèse différentielle, au contraire du modèle que nous proposons. 
Par ailleurs, l'unité forme/sens, bien que reconnue essentielle dans l'analyse linguistique, reste encore largement à élucider. La quasi-totalité des théories linguistiques se bornent en effet à enregistrer et à exploiter les corrélations formes/sens mais sans en approcher les principes internes. L'unité indivise du signe est ainsi généralement rapportée à un couplage, soit logique, soit dynamique, d'un signifiant et d'un signifié constitués pour chacun à leur propre compte. Ainsi, par exemple, dans les grammaires de contruction (Croft \& Cruse, 2004) (en prolongement des grammaires cognitives de Langacker (Langacker, 2008)), l'unité intégrée de la forme de mot et de sa signification résulte d'un processus d'association et de stockage (entrenchment) d'une routine de co-actualisation (de la forme et du sens) basée sur la réitération de cooccurrences et non pas sur une "interpénétration" (de la forme et du sens). Forme de mot et signification sont donc élaborées préalablement et indépendamment l'une de l'autre.

\section{VI.3 - Les spécificités du modèle phénoménologique, morphodynamique et structuraliste}

Nous rappellerons ici les caractéristiques fondamentales du modèle que nous proposons, caractéristiques présentées dans l'introduction. Le modèle phénoménologique, morphodynamique et structuraliste (PMS) se fonde sur trois socles : 1) l'unité constitutive a priori de la forme et du sens (versus une approche intégrative a posteriori de la forme et du sens, comme dans les grammaires cognitives); 2) la différentialité du sens reprise de Saussure, rendue par une approche qualitative des systèmes dynamiques; et 3) une approche phénoménologique qui reconnait des phases de conscience verbale intermédiaires entre le son et le sens et qui soutient une approche conscientielle (et donc intentionnelle) de la linguistique. Notre modèle est donc dynamique, morphologique et intentionnel.

Par comparaison avec d'autres approches dynamiques non morphologiques (Victorri, Culioli), nous n'envisageons pas des valeurs sémantiques définies comme des portions d'un espace sémantique mais des valeurs différentielles: nous ne nous intéressons pas aux dynamiques comme modalités de détermination d'un sens actuel et positif dans un référentiel posé, mais au type qualitatif des dynamiques (leurs formes) et à leur distribution. C'est cette distribution qui s'actualise dans l'espace de signification sous forme des frontières.

Ainsi dans l'approche morphodynamique, l'espace sémantique ne dispose au départ d'aucun référentiel. C'est dans une logique et un processus d'émergence que se constitue la structure différentielle (frontières catastrophistes) qui catégorise le continuum sémantique en identités oppositives de sens. Ainsi, la forme de mot contrôle l'émergence d'un sens qui ne lui pré-existe pas. On ne peut donc concevoir l'un sans l'autre.

Par comparaison aux grammaires cognitives, le caractère indivisible du signe relève de la phénoménalisation de la langue. Ce signe peut être approché d'une part, grâce à sa caractérisation formelle et d'autre part, grâce à sa phénoménalité. De ce fait notre approche est intentionnelle. En ce sens la description phénoménologique de Husserl en conscience primaire et 
thématique relève d'une problématique de l'expérience de ce que c'est que de "rencontrer" un signe. Dès lors cette rencontre se décrit comme une expérience consciente dont il conviendrait d'expliciter la part pré-réflexive de manière détaillée par exemple par des entretiens d'explicitation (Balzani et al., 2013), ce qui est un des projets de travail de ce programme phénoménologique.

\section{VII - LA N400 : LE TRAITEMENT DU SENS VU PAR L'ÉLECTROPHYSIOLOGIE}

Les neurocientifiques ne disposent pas de beaucoup d'outils pour analyser finement un phénomène aussi complexe que le langage. L'électrophysiologie permet néanmoins de suivre avec une très grande précision le décours temporel des processus impliqués dans la perception et la compréhension du langage. En particulier, la méthode des Potentiels Évoqués $(\mathrm{PEs})^{18}$, dérivée de l'électroencéphalographie (EEG) a permis de montrer que la composante N400 est un indice intéressant pour les études en neurolinguistique.

\section{VII.1 - Le potentiel évoqué N400}

En neurolinguistique, l'étude des composantes des potentiels évoqués comme la composante N400 s'est développée depuis le début des années 1980 (voir pour synthèse plus détaillée Kutas et al., 2006; Kutas \& Federmeier, 2011 ; Piotrowski, 2009 ; Cermolacce et al., 2013). Initialement décrite dans la modalité visuelle par Kutas \& Hillyard (1980), mais obtenue également en modalité auditive (Holcomb \& Neville, 1990; Vion-Dury et al, 2015), la composante N400 est une négativité débutant chez les sujets sains vers $250 \mathrm{~ms}$ après le début de la présentation d'un item, et dont l'amplitude maximale se situe vers $400 \mathrm{~ms}$ sur la région centro-postérieure droite du cerveau (figure 4).

Les variations de cette composante négative sont communément observées pour un mot cible lors de manipulations du contexte sémantique, que ce contexte consiste en une présentation initiale d'un mot amorce (amorçage sémantique par paires de mots), d'une phrase entière ou d'un corpus de quelques phrases. Ici, les notions de contexte et d'associations sémantiques font référence à un modèle (en pratique un modèle connexionniste distribué) de réseau sémantique généralement admis en neurolinguistique, à savoir le réseau neural où les concepts sont représentés par les nœuds du réseau, et les associations sémantiques par les liens (activateurs ou inhibiteurs) entre ces nœuds. Lorsqu'un concept est activé (par un autre mot, par un stimulus), l'activation se propage aux concepts attenants, et s'estompe progressivement au fil d'associations de plus en plus lointaines (Collins \& Loftus, 1975; Kutas ; Federmeier, 2000 ; Kutas \& Federmeier, 2011 ; Whitney et al., 2011).

\footnotetext{
${ }^{18}$ Rappelons succinctement que les potentiels évoqués sont des variations de voltage (ou d'intensité) survenant dans le signal électroencéphalographique (EEG) d'un processus faisant partie d'une tâche perceptive, motrice ou cognitive (potentiels évoqués cognitifs ou «Event-Related Potentials », ERP (Vion-Dury et al., 2015, ch. 5). Les potentiels évoqués cognitifs (ERP) présentent des latences plus tardives que les potentiels sensori-moteurs tels qu'on les utilise par exemple en neurologie. Tout stimulus est suivi de réponses à courte latence, et de réponses à longue latence (>100 $\mathrm{ms}$ ) correspondant au traitement cognitif dans les aires corticales associatives. Les aspects méthodologiques spécifiques aux potentiels évoqués pourront être étudiés dans (Luck, 2005).
} 


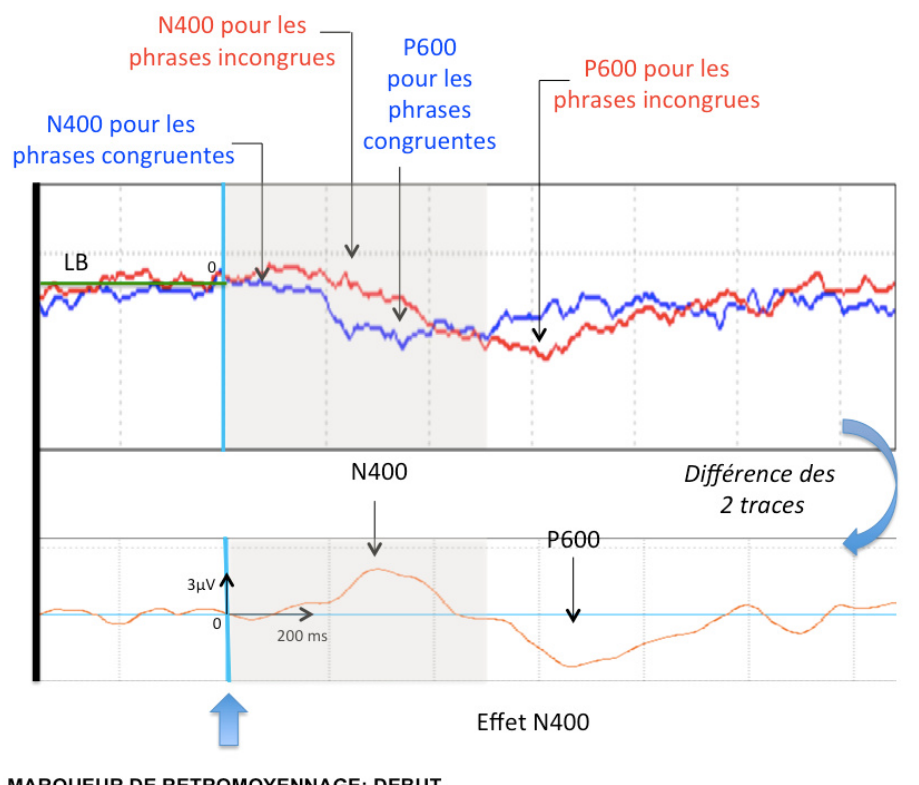

NNAGE: DEBUT

DU DERNIER MOT DE CHAQUE PHRASE

Figure 4

Potentiels N400 obtenus à partir d'un ensemble de 60 phrases dont le mot final est congruent par rapport au contexte et de 60 phrases dont le dernier mot est incongru dans le contexte. Le marqueur de rétromoyennage est placé au début de ce dernier mot (flèche épaisse). La zone de signal située avant ce marqueur est utilisée pour définir la ligne de base (LB).On obtient un tracé pour les phrases congruentes et un tracé pour les phrases incongrues. Chaque tracé contient une négativité à $400 \mathrm{~ms}$. La différence des deux traces conduit à l'effet N400, lequel exprime l'effet de l'incongruité sur les processus cognitifs (la fenêtre temporelle de la N400 est en grisé). La N400 est suivie d'une onde positive dite P600 d'interprétation discutée: selon les auteurs, elle serait liée à l'intégration des traitements sémantique, syntaxique et orthographique, ou bien à la mise à jour générale du contexte, ou bien encore reflèterait des mécanismes d'analyse et de vérification (Cermolacce et al. 2013)

Dans son interprétation la plus large, la N400 est reconnue pour manifester au plan neurophysiologique un traitement de nature sémantique (Pour revue voir (Lau et al, 2008 ; Kutas \& Federmeier, 2011)). On observe en effet que son amplitude est corrélée à la difficulté d'intégration d'un mot à son contexte sémantique.

Précisément, l'amplitude de cette négativité est augmentée lorsqu'un mot est incongru, inattendu ou non adéquat par rapport au contexte linguistique (Kutas \& Federmeier, 2011).

D'autres facteurs peuvent aussi intervenir moduler la composante N400 en dehors d'un contexte sémantique: 1) le traitement d'un mot isolé génère une onde N400, dont l'amplitude est inversement corrélée à la fréquence d'usage du mot (Van Petten \& Kutas, 1991) ; 2) lorsque deux phrases, toutes deux congruentes, diffèrent par la prédictibilité du mot final, le mot sémantiquement 
le moins prédictible est associé à une composante N400 plus ample que le mot le plus prédictible ( Kutas \& Hillyard, 1984) ; 3) la N400 est plus ample pour un mot concret (plutôt qu'abstrait) ce qui est interprété comme lié au fait qu'un mot concret appartient à un réseau sémantique plus dense, plus important qu'un mot abstrait (Paller et al, 1987). Il en est de même pour un mot peu typique d'une catégorie sémantique: par exemple, goyave est un mot peu typique de la catégorie sémantique des fruits car si on demande à un sujet (en France métropolitaine) d'énoncer les noms de fruits qui lui viennent à l'esprit, le mot goyave sera moins probable que le mot poire ou pomme; 4) enfin, la composante N400 est plus ample lors de la première présentation d'un mot qu'après sa répétition (Stuss et $a l, 1988$; Besson et $a l, 1992$ )

Nous devons noter qu'on ne peut associer de manière simple les potentiels évoqués (qui sont la conséquence de l'activité d'un réseau important de neurones intégrant des voies excitatrices et inhibitrices dans le temps (Luck, 2005)), à un rôle linguistique unique. La composante N400 n'est d'ailleurs pas spécifiquement linguistique (elle est suscitée par des images incongrues, des équations fausses en arithmétique, etc.). Mais son amplitude et sa latence varient en fonction de différents facteurs linguistiques (fréquence d'occurrence des mots dans la langue, répétition, mots concrets/abstraits, etc.) et c'est pourquoi elle peut être utilisée pour tester des hypothèses en psycholinguistique.

\section{VII.2 - Signification proposée de la N400 par les modèles fonctionnalistes}

La composante N400 obtenue dans une tâche cognitive donnant lieu à un «traitement» sémantique a reçu 4 principales interprétations : 1) comme signature neurobiologique d'un processus d'intégration sémantique ; 2) comme marqueur de l'incongruité sémantique d'une unité par rapport à son contexte ; 3) comme mesure du degré d'anticipation d'une unité sémiolinguistique ${ }^{19}$; et 4) comme signature neurobiologique d'un processus d'accès lexical (Piotrowski, 2009). Les interprétations 1, 2 et 3 se recouvrent et qualifient la N400 comme marqueur du coût neurocognitif de construction d'une représentation sémantique globale et synthétique, cette construction étant d'autant plus aisée que les termes présentés sont congruents ou anticipés. L'interprétation 4 concerne les composantes N400 obtenues en réponse à des termes isolés, présentés hors contexte phrastique ou sans amorçage, donc hors tout rapport de congruité.

La N400 peut donc être considérée soit comme marqueur d'intégration (en lien avec les processus de détection de l'incongruité du mot par rapport au contexte), soit comme un marqueur d'accès lexical (cas des mots isolés) (Van Petten \& Luka, 2012). Toutefois, dans une problématique des réseaux (Ferrand, 1994), la qualification de la N400 en termes d'intégration peut se comprendre en termes d'accès lexical. Le processus d'intégration peut être vu comme une somme d'accès aux différentes unités composant le complexe

\footnotetext{
${ }^{19}$ On doit distinguer entre «un marqueur de l'incongruité sémantique d'une unité par rapport à son contexte » et une «mesure du degré d'anticipation d'une unité sémiolinguistique ». En effet on peut avoir des mots également congrus à leurs contextes mais plus ou moins anticipés (mesure par la « cloze probability »).
} 
sémiotique et se trouvant pour chacun conditionné par ce contexte, favorablement ou au contraire comme empêchant l'intégration. Le mécanisme d'intégration s'avère ainsi réductible à des opérations interactives d'accès au lexique. D'autres auteurs, plus récemment, proposent que la N400 soit le témoin d'une facilitation d'accès lexical, plutôt que porteuse d'un rôle purement intégratif, avec un réseau de représentations lexicales amodales lié à des représentations sémantiques présentes dans tout le cortex et intégrés dans le gyrus temporal médian (Lau et al., 2008).

La majorité des interprétations des variations d'amplitude et/ou de latence de la N400 se trouve formulée dans le contexte du paradigme fonctionnaliste, auquel appartient la neurolinguistique dans son courant majoritaire (VionDury, 2007) et tel qu'il a été défini plus haut.

Cependant comme nous l'avons vu dans le paragraphe VI.1, il existe une évolution très rapide des modèles neurolinguistiques et linguistiques. C'est ainsi que certains modèles, plutôt modularistes, soulignent le rôle spécifiquement joué par certaines structures cérébrales (Friederici, 2012). Différents modèles d'activation interactive ont été également proposés pour dépasser les limites de la conception modulariste (pour revue voir (Carreiras, Armstrong, Perea, \& Frost, 2014). À titre d'exemple, le PDP model (Parallel Distributed Processing) a été proposé pour expliquer les tâches de décision lexicale dans la lecture visuelle et expliquer les variations d'intensité de la N400 à partir des sous composantes du potentiel; ce modèle semble généralisable à d'autres opérations cognitives de haut niveau (Laszlo \& Plaut, 2012). Ces modèles connexionnistes considèrent que les différentes zones cérébrales impliquées dans le langage interagissent entre elles dans des réseaux dynamiques. Dans ces modèles, la fonctionnalité d'une région est déterminée par le réseau de structures cérébrales auxquels elle appartient à un moment donné (Hagoort, 2014). Cette conception est globalement en accord avec celle de Friederici et Singer qui soulignent que des réseaux complexes d'assemblée de neurones auto-organisés et flexibles organisés grâce à un squelette de connexions anatomiques sont connectés à d'autres réseaux ou des «hubs» permettant ainsi une intégration polymodale de la syntaxe et de la sémantique du langage (Friederici \& Singer, 2015).

\section{VII.3 - Quelques problèmes posés par les interprétations de la N400 et leurs conséquences}

L'incertitude encore patente quant à la signification de la N400 comme marqueur d'accès lexical ou, corrélativement, comme marqueur d'intégration ou d'attente sémantique nous amène à souligner quelques résultats expérimentaux intéressants, pouvant faire figure d'anomalies, mais qui pourraient être expliquées à la fois par les conceptions dominantes de la N400 et par le modèle PMS.

Les trois résultats expérimentaux en question sont :

1) Dans le cadre de tests de décision lexicale, l'amplitude de la N400 produite par des pseudomots (logatomes) est soit supérieure soit égale à celle des mots (Kutas \& Federmeier, 2000). Dans le paradigme fonctionnaliste, il est «normal » que cette amplitude soit 
supérieure, puisque le travail nécessaire pour «accéder » à un item (le rechercher et constater sa présence) au sein du lexique mental a la forme d'un parcours, et que ce parcours doit être maximal pour conclure sur l'inexistence de l'item au sein du lexique. En revanche, il est plus difficile d'expliquer l'égalité de l'amplitude de la N400 : comment le dispositif cognitif procède-t-il pour atteindre suivant une même logique d'accès au lexique et à effectivité égale (mesurée par l'amplitude des N400) une information touchant l'existence ou l'inexistence d'une forme de mot? Ces résultats contradictoires pourraient s'expliquer par: a) les propriétés intrinsèques des mots et pseudomots choisis ; b) la tâche requise par le sujet (par exemple des différences dans la consigne); c) les fluctuations du niveau d'attention (voir proposition 3 ci-dessous) ; d) un effet propre à la méthode du moyennage qui, comme le montre Luck (2005) estompe la variabilité (en durée, latence, amplitude) de chaque réponse évoquée; dès lors, selon l'importance de cette variabilité d'un sujet à l'autre, on pourrait avoir des réponses N400 différentes allant même jusqu'à définir un «style cognitif »; e) voire de la non-publication d'anomalies rencontrées dans les laboratoires (Ioannidis, 2007). Cependant les résultats contradictoires reçoivent trop facilement une explication liée à des différences méthodologiques (procédures et matériels expérimentaux) ou à la technique utilisée. Mais qu'on oublie qu'il existe un biais quasi systématique de publication de résultats concordants versus discordants en faveur des premiers (Ioannidis, 2007) laisse envisager que ce n'est pas un épiphénomène et que cela justifierait de revoir les modèles cognitifs sous-jacents, voire un changement paradigmatique, tel que nous l'envisageons. En d'autres termes cet effet de «masquage » des résultats discordants ou négatifs semble avoir un effet de «protection» du paradigme majoritaire, au prix de l'acceptation d'anomalies empiriques (Kuhn, 2008).

2) Toujours dans le cadre de tests de décision ou de reconnaissance lexicale, les non-mots ne génèrent pas de $\mathrm{N} 400$ (ou parfois une très faible) (cf. entre autres Holcomb \& Neville, 1990 ; Ziegler et $a l$, 1997 ; Kutas et al., 2006). Cette absence de réponse constitue une anomalie dans la mesure où, suivant une perspective de traitement d'information, la donnée (comme input) d'un signal graphique ou phonique doit bien faire l'objet d'un traitement initial au cours duquel sa conformité linguistique se trouve testée et suite à quoi d'autres processus peuvent être déclenchés. Ce signal devrait donc systématiquement donner lieu à une N400. Cependant, cette discordance pourrait être partiellement expliquée. Les pseudomots prononçables - génèrent une N400 (parfois même de plus grande amplitude qu'un mot inattendu) alors que les non-mots - non prononçables - sont plutôt associés à une composante de type P300 reflétant une stratégie de détection d'un stimulus inattendu non linguistique. Mais dans cette explication, d'une part, tout dépend de la forme de stockage envisagée pour le lexique (séquentielle, plus globale etc.), et, d'autre part, on pourrait aussi considérer qu'il existe 
deux stratégies de réponses pour les non-mots ? : l'une favorisant la détection d'une incongruité (P300) l'autre une stratégie de recherche sémantique (N400). On ne peut non plus éliminer le fait que la proximité temporelle des 2 composantes puisse aussi expliquer la non-visualisation de la N400 en présence d'une P300 de grande amplitude.

3) Il est connu de la littérature que l'amplitude de la N400 est fonction croissante de l'attention. On attend donc que la N400 obtenue avec les distracteurs ignorés soit inférieure à celle obtenue avec des distracteurs mémorisés. Ceci peut être testé dans un protocole de présentation de triplets (distracteur, amorce, cible) dans lequel la consigne est de chercher la relation sémantique entre amorce et cible dans deux conditions (Debruille et al., 2008; Mc Carthy et al., 1993 ; Yagamata et al., 2000) : (a) avec la consigne visant à ignorer les distracteurs, et (b) avec la consigne visant à mémoriser les distracteurs qui font ainsi l'objet d'une attention particulière. On observe que la N400 obtenue avec les distracteurs ignorés est en fait supérieure à celle obtenue avec des distracteurs mémorisés ce qui constitue l'effet inverse de l'effet attendu.

Ces quelques résultats expérimentaux n'ont pas trouvé d'explication complétement satisfaisante par les modèles neurolinguistiques actuels des neurosciences et ils pourraient de ce fait constituer des tests intéressants pour le modèle PMS.

\section{VIII - N400 ET MODÈLE MORPHODYNAMIQUE}

Dans ce paragraphe nous tenterons de voir si le modèle PMS permet une compréhension différente des processus mentaux induisant une réponse électrophysiologique de type N400 et s'il est de nature à expliquer les problèmes rencontrés dans l'interprétation de certains résultats empiriques obtenus avec ce potentiel évoqué (voir paragraphe précédent). Enfin nous décrirons rapidement le protocole électrophysiologique qui pourrait mettre à l'épreuve le modèle PMS que nous envisageons.

\section{VIII.1 - Les tests linguistiques et les aspects co-occurrents de la conscience verbale}

On peut mettre en regard ces modalisations co-occurrentes de conscience décrites dans le paragraphe $\mathrm{V}$ avec les tests linguistiques couramment utilisés dans les recherches empiriques.

- La conscience de disponibilité. Il n’y a pas, à notre connaissance, de test relevant de la conscience de disponibilité.

- La conscience d'engagement est explorée a minima par la tâche de décision lexicale : est-ce un mot (du langage) ou pas (par exemple un pseudomot), la décision lexicale pouvant prendre appui sur les autres modalisations.

- La conscience de signifié et, sous-jacente, la conscience de motif, peuvent être approchées par des tests de catégorisation sémantique. 
- Enfin la conscience de remplissement est approchée par des tâches de construction de représentations mentales.

\section{VIII.2 - Les processus induisant la N400 dans le modèle morphodynamique}

Le modèle morphodynamique nous incite à changer la signification fonctionnelle de la N400. Plutôt que marqueur de coût cognitif de constitution de sens lors d'un accès lexical, la N400 apparaîtrait comme index de l'épaisseur d'un acte intentionnel «signitif », ou si l'on préfère, de la «densité » des modalisations, instituant le phénomène du signe (le signifiant) au travers d'une visée de signification. De même qu'un accord est «polarisé » par sa note la plus haute, la conscience de son de mots est polarisée de plus en plus fortement par la co-présence morphodynamique des autres modalisations de la conscience verbale, dont l'importance relative dans un moment donné peut varier. La N400 devient alors, dans cette métaphore, un indicateur de la complétion de l'accord: si je ne joue que le do grave, la N400 sera alors minimale, le contenu de conscience ne se «remplissant » d'aucune visée vers le sens (ce do pourrait en effet faire partie d'un renversement de l'accord de fa majeur, par exemple, ou d'une sixte d'un accord de mi). C'est ce qui se passerait pour la N400 d'un logatome. Quand l'accord est complet, ce qui correspondrait à la conscience de remplissement, alors la N400 pourrait être maximale. La densification de l'accord, on le voit est indépendante d'une quelconque séquence de production des notes.

Les processus d'augmentation de la «densité des modalisations » s'effectueraient sans accès lexical et avec une notion d'intégration qui prendrait en compte non plus le système séquentiel ramifié des circuits neuronaux tels qu'ils sont habituellement décrits, mais des attracteurs se constituant et instaurant leurs identités négatives (leurs frontières) en fonction du contexte linguistique.

Sur le plan neurophysiologique, cette conception pourrait être parfaitement compatible avec la conception de la variabilité des réseaux neuronaux dans le traitement du contexte (voir plus haut paragraphe VII.2 sur la signification de la N400). Plus encore notre modèle est parfaitement cohérent avec la notion d'« unification » ou encore « intégration », notion qui traverse la linguistique depuis Humboldt jusqu'aux conceptions contemporaines (en passant notamment par Hjelmslev et Benveniste, pour les figures majeures) et reprise par Hagoort (2014). L'unification en effet se réfère à un assemblage de blocs de construction lexicale au sein de plus larges structures, modulé par le contexte et le savoir général sur le monde.

\section{VIII.3 - Vers une compréhension possible des «anomalies de la N400» par le modèle PMS?}

Puisque la conscience dispose lors de la modalisation d'engagement, d'éléments nécessaires pour distinguer un mot d'un pseudomot (existence ou non d'une fonction de contrôle), il est possible d'expliquer que, sous certaines conditions expérimentales qui ne sollicitent que certaines modalisations de la 
conscience verbale, l'amplitude de la N400 observée lors de tâches de décision lexicale soit d'intensité égale pour les mots et les pseudomots.

On a vu que la réponse N400 à des pseudomots peut être supérieure à celle des mots. On a dit aussi qu'une N400 d'amplitude supérieure pour les pseudomots se laisse aisément expliquer. Mais, si on choisit de raisonner en termes de modalisation (et d'intentionnalité), alors la réponse à un test de décision lexicale se suffit de la modalité de conscience d'engagement (au sens), et ceci quelle que soit la nature du stimulus : mot ou pseudomot. En d'autres termes, les variations d'amplitude de la N400 en réponse à des pseudomots seraient fonction de la profondeur d'accès au sens sollicitée : si le sujet reste à un palier de conscience d'engagement, par ailleurs suffisant pour répondre au test de décision lexicale, alors l'amplitude des composantes N400 aux mots et aux pseudomots ne sera pas significativement différente. Par contre, si le sujet «force» son attention vers un sens qui n'existe pas, s'il cherche à tout prix à inventer un sens, c'est-à-dire s'il «active » d'autres modalisations, il en résultera logiquement une N400 plus ample. Par ailleurs, notre approche rend aussi compte de l'inexistence de N400 pour les non-mots : puisque un non-mot, par sa morphologie, annonce d'une certain façon d'emblée qu'il ne saurait constituer un mot et donc qu'il ne saurait engager une modalisation de la conscience verbale, on comprend qu'il ne donne lieu à aucune N400.

De la même manière, il est logique que la N400 des distracteurs mémorisés (impliquant la conscience de signifiant et de motif) soit d'amplitude plus faible, que celle des items ne faisant l'objet d'aucun traitement spécifique. Simplement parce que le travail de mémorisation exigerait de la conscience d'en rester à des modalités où l'identité du signifiant est fixée. Alors que le décours naturel de la parole conduit vers l'activation de la conscience de remplissement, l'attention resterait attachée à des niveaux de conscience où le signifiant réside. La conscience verbale maintiendrait ainsi au premier plan les modalisations où s'élabore le signifiant. D'où des N400 d'amplitudes inférieures à celles des N400 émises lorsque le sujet a toute liberté d'aller aux signifiés ou, plus avant, d'élaborer des significations actuelles (voir synthèse de ces résultats dans le tableau 1 ).

Notre problématique structurale et phénoménologique peut donc rendre compte des fluctuations de la N400 suivant les configurations d'expériences qui sollicitent différentes modalisations de conscience verbale. On observe une bonne association entre l'amplitude de la N400 et la «densité » de l'engagement conscientiel. Ainsi, plus généralement, la N400 observée avec des mots concrets est supérieure à celle obtenue pour des mots abstraits parce que leur traitement engage un remplissement plus riche); de même, la N400 enregistrée lorsqu'on demande aux sujets de construire une image mentale est supérieure à celle obtenue lorsqu'on s'en tient aux significations ; ou encore, la N400 observée dans des tests de catégorisation sémantique (qui sollicitent l'accès au sens) est supérieure à celle obtenue lors de test de décision lexicale qui se satisfont d'une conscience d'engagement. 


\begin{tabular}{|c|c|c|}
\hline $\begin{array}{c}\text { Résultat empirique } \\
\text { observé }\end{array}$ & $\begin{array}{l}\text { Interprétation dans } \\
\text { le modèle standard } \\
\text { (N400 comme coût cognitif } \\
\text { d'accès au lexique) }\end{array}$ & $\begin{array}{l}\text { Explication par le modèle } \\
\text { PMS. (La N400 marqueur } \\
\text { de densité de la } \\
\text { conscience verbale) }\end{array}$ \\
\hline $\begin{array}{l}\text { L'amplitude de la N400 } \\
\text { produite par des } \\
\text { pseudo-mots } \\
\text { (logatomes) peut ne } \\
\text { pas être différente de } \\
\text { celle des mots. }\end{array}$ & $\begin{array}{l}\text { Cela pourrait dépendre des } \\
\text { propriétés intrinsèques des } \\
\text { mots et pseudo-mots choisis, } \\
\text { de la tâche requise du sujet, } \\
\text { des fluctuations du niveau } \\
\text { d'attention, d'un effet du } \\
\text { moyennage entre items. }\end{array}$ & $\begin{array}{l}\text { La conscience d'engagement } \\
\text { est la même qu'il s'agisse } \\
\text { d'un mot ou d'un pseudo- } \\
\text { mot (logatome). Si le sujet } \\
\text { " force » son attention vers } \\
\text { un sens qui n'existe pas, } \\
\text { (" activation » d'autres } \\
\text { modalisations), la N400 sera } \\
\text { plus ample. }\end{array}$ \\
\hline $\begin{array}{l}\text { Les non-mots ne } \\
\text { génèrent pas de N400 } \\
\text { (ou parfois une très } \\
\text { faible) }\end{array}$ & $\begin{array}{l}\text { Les non-mots - non } \\
\text { prononçables - sont plutôt } \\
\text { associés à une composante } \\
\text { de type P300. Il pourrait } \\
\text { exister deux stratégies de } \\
\text { réponses : I'une favorisant la } \\
\text { détection d'une incongruité } \\
\text { (P300) l'autre une stratégie } \\
\text { de recherche sémantique } \\
\text { (N400) }\end{array}$ & $\begin{array}{l}\text { Pas de modalisation } \\
\text { d'engagement donc pas de } \\
\text { N400. }\end{array}$ \\
\hline $\begin{array}{l}\text { L'amplitude de la N400 } \\
\text { obtenue dans une } \\
\text { tâche nécessitant une } \\
\text { attention faible est } \\
\text { supérieure à celle } \\
\text { obtenue au cours d'une } \\
\text { tâche nécessitant une } \\
\text { attention plus intense. }\end{array}$ & $\begin{array}{l}\text { L'amplitude de la N400 } \\
\text { devrait être une fonction } \\
\text { croissante de l'attention, et } \\
\text { donc l'amplitude de la N400 } \\
\text { obtenue avec les distracteurs } \\
\text { ignorés devrait être } \\
\text { inférieure : c'est une } \\
\text { anomalie. }\end{array}$ & $\begin{array}{l}\text { Parce que orientée vers le } \\
\text { signifiant, l'attention } \\
\text { " bloque » la conscience } \\
\text { verbale dans la conscience } \\
\text { de signifiant. Lors de } \\
\text { l'absence de tâche } \\
\text { attentionnelle, l'activation } \\
\text { de la conscience de } \\
\text { remplissement conduit à } \\
\text { une N400 plus ample. }\end{array}$ \\
\hline
\end{tabular}

\section{Tableau 1}

Synthèse des difficultés d'interprétation des anomalies de la N400 dans le modèle d'accès lexical et de la compréhension possible de ces anomalies dans le modèle PMS.

Notre problématique structurale et phénoménologique peut donc rendre compte des fluctuations de la N400 suivant les configurations d'expériences qui sollicitent différentes modalisations de conscience verbale. On observe une bonne association entre l'amplitude de la N400 et la «densité » de l'engagement conscientiel. Ainsi, plus généralement, la N400 observée avec des mots concrets est supérieure à celle obtenue pour des mots abstraits parce que leur traitement engage un remplissement plus riche); de même, la N400 enregistrée lorsqu'on demande aux sujets de construire une image mentale est 
supérieure à celle obtenue lorsqu'on s'en tient aux significations ; ou encore, la N400 observée dans des tests de catégorisation sémantique (qui sollicitent l'accès au sens) est supérieure à celle obtenue lors de test de décision lexicale qui se satisfont d'une conscience d'engagement.

\section{VIII.4 - Vérification de l'applicabilité modèle morphodynamique en neurolinguistique}

Notre approche doit être éprouvée dans ce qu'elle comporte de spécifique, à savoir à travers ses composantes descriptives relevant de la conscience verbale. C'est ainsi au niveau de la conscience de disponibilité que l'on focalisera notre attention, à savoir la conscience d'un item présent en tant que simplement disposé à participer d'une construction langagière. En effet, parmi les différentes modalisations de conscience verbale qui ne trouvent pas de répondant dans les autres cadres théoriques, notamment fonctionnalistes, c'est celle de la «disponibilité » qui semble la plus aisée à établir expérimentalement et à explorer contrastivement. D'une part, cette modalisation de «disponibilité » se situe au palier le plus «profond » (ou au moins dense) de l'acte sémiolinguistique ; elle pourrait donc être atteinte en faisant, par certaines conditions expérimentales, «tendre vers zéro »la conscience verbale. D'autre part, alors que les autres modalisations établissent des distinctions nouvelles dans les niveaux d'analyse reconnus par les approches fonctionnalistes, la conscience de disponibilité n'a pas d'équivalent. Elle s'offre donc naturellement comme terrain d'une expérience «cruciale». C'est donc cette modalisation de conscience qu'il nous faut approcher, dévoiler et étudier expérimentalement.

Pour ce faire, nous avons conçu un protocole, dit de WordSpotting Généralisé, ainsi nommé parce qu'il croise les protocoles de «Wordspotting » et de «flux syllabiques». Dans ses grandes lignes, il s'agira de soumettre les sujets à des flux de syllabes aléatoires au sein desquels se trouvent plongés de « vrais » mots. Les sujets seront invités à effectuer des tâches sollicitant diverses modalisations de la conscience verbale. Notre hypothèse est que l'attitude d'écoute des syllabes aléatoires se limitera à une conscience de disponibilité car d'une part, la présence possible de vrais mots doit garder l'intentionnalité sémiolinguistique en alerte, comme horizon d'appréhension, et, d'autre part, la connaissance du caractère aléatoire des suites de syllabes invite à ne pas les prendre aussitôt pour des mots, mais à les garder en réserve au cas où elles pourraient faire mot avec d'autres syllabes ultérieures - et c'est bien là une conscience de disponibilité. Une expérimentation réalisant un enregistrement la N400 lors de l'apparition de mots dans un flux de syllabes lors de la variation du type de conscience engagée dans des tâches différentes (par exemple demander au sujet de se mettre dans une attention flottante et contraster avec une tâche de décision lexicale) serait de nature à tester cette hypothèse.

\section{IX - CONCLUSION}

Les modèles linguistiques sont complexes, sans cesse en évolution, et s'enrichissent des avancées mathématiques (comme les attracteurs ou les systèmes complexes). Il en est de même pour les modèles de la 
neurophysiologie ou des neurosciences cognitives, qui eux aussi se complexifient progressivement et se rapprochent de l'implémentation possible de processus formels dans des systèmes nerveux, sous la forme de propositions neurocomputationnelles. Un tout récent exemple, mais qui récapitule tout un courant conceptuel, est la proposition d'une psychologie fondée sur des processus statistiques de nature quantique plutôt que bayésienne (Bruza et $a l$, 2015).

Le modèle morphodynamique développé dans cet article ne s'oppose pas à ces propositions théoriques des neurosciences les plus avancées car il utilise les notions de systèmes dynamiques et d'attracteurs. C'est un modèle connexionniste, distribués, interactif et dynamique. Cependant il se différencie par deux points majeurs. D'une part il postule l'unité constitutive et indivise du signe, ce qui relève d'un point de vue phénoménologique d'une problématique relative à l'origine de celui-ci et à l'acceptation que le signifiant et le signifié présentent le même caractère primordial, aucun n'étant à l'origine de l'autre. En ce sens cette conception s'éloigne d'une position physicaliste et réductrice qui sous-tend, même avec de nombreuses nuances, la théorie de l'accès lexical (Forster \& Chambers, 1973). D'autre part, il introduit dans la donation de sens, la conscience, non pas dans un processus séquentiel d'émergence sous la forme d'une conscience verbale de plus en plus complexe, mais sous la forme de la coprésence de ses modalisations, en des proportions diverses selon le niveau de compréhension requis. Notre approche pourrait être en partie implémentée dans des modèles neurophysiologiques assez proches de ceux de Hagoort, en particulier par le biais de la notion d'unification sous-tendue par des réseaux et des nœuds éminemment variables, contextuels et flexibles, tout autant qu'étendus (Hagoort, 2014). Pour autant le modèle d'Hagoort ne prend pas à son compte les traditions phénoménologiques et saussuriennes, et ne considère pas la problématique de la conscience verbale.

L'importance donnée à la phénoménologie comme fondement de notre réflexion sémiotique, et le projet de réaliser une validation secondaire, empirique, du modèle PMS sous la forme de l'étude des variations de la composante $\mathrm{N} 400$ procède d'une approche neurophénoménologique que Gallagher nomme «front loaded phenomenology», c'est-à-dire une phénoménologie qui prend place avant toute expérimentation empirique, tout simplement parce qu'elle la fonde dans l'expérience subjective (Gallagher \& Brøsted Sørensen, 2006), comme l'a pensé Husserl (Husserl, 1985). Car, si chez Husserl la phénoménologie semble très théorique, elle provient de l'expérience subjective en quelque sorte décantée, comme le montrent par exemple nombre de paragraphes de «La synthèse passive » (Husserl, 1998). Ainsi, dans notre approche de la neurophénoménologie, ce terme créé par Varela (Depraz et al., 2011) nous semble devoir finalement s'écrire neuroPhénoménologie, pour signifier le caractère fondateur de la phénoménologie et donc de l'expérience subjective.

Il faudrait ainsi aller encore plus loin et décrire finement ce qui se produit lors de l'accès au sens d'un mot, du point de vue du vécu conscient. Outre le projet de la mise à l'épreuve du modèle PMS par la composante N400 que nous avons détaillé plus haut, nous avons initié une étude utilisant le matériel linguistique réalisé et portant sur l'explicitation des vécus de conscience 
verbale (Vermersch, 2012 ; Balzani et al., 2013) de sujets à qui on a présenté ce matériel. Les données ne sont pas encore analysées, mais de manière très préliminaire, il apparaît lors de l'écoute du WordSpotting Généralisé, que d'une part les sujets modalisent leur conscience de manière très différente que lors de l'écoute d'un discours «classique » et l'orientent vers une attention flottante proche de l'hypnose et que, d'autre part, la survenue de mots dans le flux module cette attention flottante de manière différente s'il s'agit de mots à deux ou trois syllabes. Ainsi ce second type de protocole, typiquement phénoménologique est un autre moyen de mettre à l'épreuve le modèle PMS, complémentaire de la perspective neurophysiologique.

\section{RÉFÉRENCES}

Balzani, C., Micoulaud-Franchi, J.-A., Yunez, N., Fagot, A., Mariaud A.-S., Chen, C.Y. \& Vion-Dury, J. (2013). L'accès aux vécus pré-réflexifs. Quelles perspectives pour la médecine en général et la psychiatrie en particulier? Annales Médico-psychologiques, revue psychiatrique, 171(2), 118-127.

Bassett, D.S. \& Gazzaniga, M.S. (2011). Understanding Complexity in the Human Brain. Trends in Cognitive Sciences, 15, 200-209.

Benveniste, E. (1966). Problèmes de linguistique générale, tome 1. Paris, Gallimard.

Benveniste, E. (1973). Problems in General Linguistics. Coral Gables, Fla., University of Miami Press.

Benveniste, E. (1976). Problèmes de linguistique générale, tome 2. Paris, Gallimard.

Bitbol, M. (2008). Mécanique quantique: Une introduction philosophique. Paris, Flammarion.

Bompard-Porte, M. \& Thom, R. (1994). Passion des formes : dynamique qualitative sémiophysique et intelligibilité : à René Thom. Paris, ENS Éditions.

Bruza, P.D., Wang, Z. \& Busemeyer, J.R. (2015). Quantum Cognition: a New Theoretical Approach to Psychology. Trends in Cognitive Sciences, 19 (7), 383393.

Cadiot, P. \& Visetti, Y.-M. (2001). Pour une théorie des formes sémantiques: motifs, profils, thèmes. Paris, Presses Universitaires de France.

Carreiras, M., Armstrong, B.C., Perea, M. \& Frost, R. (2014). The What, When, Where, and How of Visual Word Recognition. Trends in Cognitive Sciences, 18(2), 90-98.

Cermolacce, M., Micoulaud-Franchi, J.-A., Faugere, M., Naudin, J., Besson, M. \& Vion-Dury, J. (2013). Électrophysiologie et vulnérabilité schizophrénique: la composante N400 comme endophénotype candidat? Neurophysiologie Cliniquel Clinical Neurophysiology, 43, 81-94

Collins, A.M. \& Loftus, E.F. (1975). A Spreading-Activation Theory of Semantic Processing. Psychological Review, 82(6), 407-428.

Croft, W. \& Cruse, D.A. (2004). Cognitive Linguistics. Cambridge University Press.

Culioli, A. (2000). Pour une linguistique de l'énonciation. Formalisation et opérations de repérages. Paris, Ophrys.

Culioli, A. (1991). Pour une linguistique de l'énonciation: Opérations et représentations, tome 1. Paris, Ophrys.

Debruille, J.B., Ramirez, D., Wolf, Y., Schaefer, A., Nguyen, T.-V., Bacon, B.A., Renoult, L. \& Brodeur, M. (2008). Knowledge Inhibition and N400: a Within- and a Between-subjects Study with Distractor Words. Brain Research, 1187, 167-183.

Depraz, N. (2002). La conscience: Approches croisées, des classiques aux sciences cognitives. Paris, Armand Colin.

Depraz, N., Varela, F.J. \& Vermersch, P. (2011). À l'epreuve de l'experience : pour une pratique phénoménologique. Bucarest, Zeta Books. 
Dupuy, J.-P. (2000). Les savants croient-ils en leurs théories? une lecture philosophique de l'histoire des sciences cognitives. Paris, Éditions INRA.

Dupuy, J.-P. (2013). Aux origines des sciences cognitives. Paris, La Découverte.

Ferrand, L. (1994). Accès au lexique et production de la parole: un survol. L'année psychologique, 94(2), 295-311.

Forster, K.I. \& Chambers, S.M. (1973). Lexical Access and Naming Time. Journal of Verbal Learning and Verbal Behavior, 12(6), 627-635.

Friederici, A.D. (2012). The Cortical Language Circuit: from Auditory Perception to Sentence Comprehension. Trends in Cognitive Sciences, 16(5), 262-268.

Friederici, A.D. \& Singer, W. (2015). Grounding Language Processing on Basic Neurophysiological Principles. Trends in Cognitive Sciences, 19(6), 329-338.

Gallagher, S. \& Brøsted Sørensen, J. (2006). Experimenting with Phenomenology. Consciousness and Cognition, 15(1), 119-134.

Gardner, H. (1993). Histoire de la révolution cognitive. La nouvelle science de l'esprit. Paris, Payot.

Godel, R. (1957). Les Sources manuscrites du Cours de linguistique généralee de F. de Saussure. Genève, Droz.

Granger, G.-G. (1979). Langages et épistémologie. Paris, Klincksieck.

Guillaume, G. (1991). Langage et science du langage. Paris, Nizet.

Hagoort, P. (2014). Nodes and Networks in the Neural Architecture for Language: Broca's Region and Beyond. Current Opinion in Neurobiology, 28, 136-141.

Hjelmslev, L. (1971). Essais linguistiques. Paris, Éditions de Minuit.

Hjelmslev, L. (2000). Prolégomènes à une théorie du langage, suivi de la structure fondamentale du langage. Paris, Éditions de Minuit.

Holcomb, P.J. \& Neville, H.J. (1990). Auditory and Visual Semantic Priming in Lexical Decision: A Comparison Using Event-related Brain Potentials. Language and Cognitive Processes, 5(4), 281-312

Husserl, E. (1985). Idées directrices pour une phénoménologie. Paris, Gallimard.

Husserl, E. (1998). De la synthèse passive. Grenoble, Jérôme Millon.

Husserl, E. (2000). Leçons sur la théorie de la signification. Paris, Librairie Philosophique Vrin.

Husserl, E. (2003). Recherches logique, tome 1: Prolégomènes à la logique pure. Paris, Presses Universitaires de France.

Ioannidis, J.P.A. (2007). Why Most Published Research Findings are False: Author's Reply to Goodman and Greenland. PLoS medicine, 4(6), e215.

Jakobson, R. (2003). Essais de linguistique générale. Paris, Éditions de Minuit.

James, W. (2003). Précis de psychologie. Paris, Les Empêcheurs de Penser en Rond.

Janet, P. (2005). L'automatisme psychologique: Essai de psychologie expérimentale sur les formes inférieures de l'activité humaine. Paris, L'Harmattan.

Klinkenberg, J.-M. (2000). Précis de sémiotique générale. Paris, Seuil.

Kuhn, T.-S. (2008). La structure des révolutions scientifiques. Paris, Flammarion.

Kutas, M. \& Federmeier K.D. (2000). Electrophysiology Reveals Semantic Memory Use in Language Comprehension. Trends in Cognitive Sciences, 4(12), 463-470.

Kutas, M, \& Hillyard, S.A. (1984). Brain Potentials During Reading Reflect Word Expectancy and Semantic Association. Nature, 307(5947), 161-163.

Kutas, M. \& Federmeier, K.D. (2011). Thirty Years and Counting: Finding Meaning in the N400 Component of the Event-related Brain Potential (ERP). Annual Review of Psychology, 62, 621-647.

Kutas M., Van Petten, C.K. \& Kluender, R. (2006). Chapter 17 - Psycholinguistics Electrified II (1994-2005). In M.J. Traxler \& M.A. Gernsbacher (éds.), Handbook of Psycholinguistics (Second Edition) (pp. 659-724). London, Academic Press.

La Mantia, F. (2014). Antoine Culioli. L'arco e la freccia. Scritti scelti. Sul lessico della linguistica di Culioli. Bologna, Il Mulino. 
Langacker, R.W. (2008). Cognitive Grammar: A Basic Introduction. Oxford University Press.

Laszlo, S. \& Plaut, D.C. (2012). A Neurally Plausible Parallel Distributed Processing Model of Event-Related Potential Word Reading Data. Brain and Language, 120(3), 271-281.

Lau, E.F., Phillips, C. \& Poeppel, D. (2008). A Cortical Network for Semantics: (de)constructing the N400. Nature Reviews. Neuroscience, 9(12), 920-933.

Luck, S.J. (2005). An Introduction to the Event-related Potential Technique. Cambridge, Mass., The MIT Press.

Mahmoudian, M. (2013). Arbitraire et différentiel chez Saussure, portée et limites. La linguistique, 48(2), 3-26.

McCarthy, G. \& Nobre, A.C. (1993). Modulation of Semantic Processing by Spatial Selective Attention. Electroencephalography and Clinical Neurophysiology, 88(3), 210-219.

Nicolescu, B. (2012). Nous, la particule et le monde. Fernelmont, EME.

Paty, M. (2003). La physique du XX siècle. Les Ulis, EDP Sciences.

Petitot-Cocorda, J. (1985). Morphogenèse du sens, tome I. Paris, Presses Universitaires de France.

Petitot, J. (1993). Phénoménologie naturalisée et morphodynamique : la fonction cognitive du synthétique a priori. Intellectica, 17, 79-126.

Petitot, J. (2008). Neurogéométrie de la vision: modèles mathématiques et physiques des architectures fonctionnelles. Paris; Éditions de l'École Polytechnique.

Petitot-Cocorda, J. (1997). Physique du sens: De la théorie des singularités aux structures sémio-narratives. Paris, Éditions du CNRS.

Petitot-Cocorda, J. (2009). Les catastrophes de la parole, de Roman Jakobson à René Thom. Paris, Maloine.

Piotrowski, D. (1997). Dynamique et structures en langue. Paris, Éditions du CNRS.

Piotrowski, D. (2009). Phénoménalité et objectivité linguistiques. Paris, Honoré Champion.

Piotrowski, D. (2013). L'opposition Sémiotique/Sémantique comme articulation de la conscience verbale. Versus - Quaderni di Studi Semiotics, (117), 27-52.

Saussure, F. de. (1995). Cours de linguistique générale. Paris, Payot.

Thom, R. (1977). Stabilité structurale et morphogénèse. Paris, Interéditions

Van Petten, C, \&Kutas, M. (1991). Influences of Semantic and Syntactic Context on Open- and closed-class words. Memory and Cognition, 19(1), 95-112.

Van Petten, C. \& Luka, B.J. (2012). Prediction During Language Comprehension: Benefits, Costs, and ERP Components. International Journal of Psychophysiology: Official Journal of the International Organization of Psychophysiology, 83(2), 176190.

Varela, F.J., Thomson, E. \& Rosch, E. (1993). L'inscription corporelle de l'esprit: sciences cognitives et expérience humaine. Paris, Éditions du Seuil.

Vermersch, P. (2012). Explicitation et phénoménologie. Paris, Presses Universitaires de France.

Victorri, B. \& Fuchs, C. (1992). Construire un espace sémantique pour représenter la polysémie d'un marqueur grammatical: l'exemple de «encore». Linguisticae investigationes: Revue internationale de linguistique française et de linguistique générale, 16(1), 125-153.

Victorri, B. \& Fuchs, C. (1996). La polysémie. Paris,

Vion-Dury, J. (2007). Entre mécanisation et incarnation: réflexion sur les neurosciences cognitives fondamentales et cliniques. Revue de neuropsychologie, 17(4), 293-361.

Vion-Dury J., Balzani, C. \& Micoulaud-Franchi, J.-A. (2015). Neurophysiologie clinique en psychiatrie: Pratique diagnostique et thérapeutique. Paris, Elsevier Masson. 
Whitney, C., Kirk, M., O’Sullivan, J., Lambon Ralph, M.A. \& Jefferies, E. (2011). The neural Organization of Semantic Control: TMS Evidence for a Distributed Network in Left Inferior Frontal and Posterior Middle Temporal Gyrus. Cerebral Cortex, 21(5), 1066-1075.

Yamagata, S., Yamaguchi, S. \& Kobayashi, S. (2000). Event-related Evoked Potential Study of Repetition Priming to Attended and Unattended Words. Brain Research. Cognitive Brain Research, 10(1-2), 167-171.

Ziegler, J.C., Besson, M., Jacobs, A.M., Nazir, T. A., \& Carr, T.H. (1997). Word, Pseudoword, and Nonword Processing: a Multitask Comparison Using Eventrelated Brain Potentials. Journal of Cognitive Neuroscience, 9(6), 758-775. 